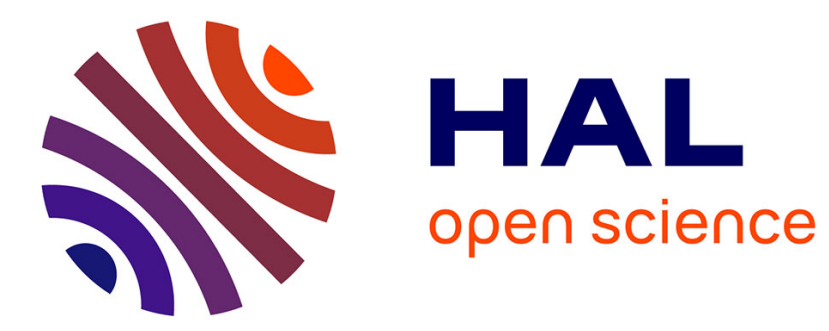

\title{
Multimodal vibration damping of a plate by piezoelectric coupling to its analogous electrical network
}

Boris Lossouarn, Jean-François Deü, Mathieu Aucejo, Kenneth A. Cunefare

\section{To cite this version:}

Boris Lossouarn, Jean-François Deü, Mathieu Aucejo, Kenneth A. Cunefare. Multimodal vibration damping of a plate by piezoelectric coupling to its analogous electrical network. Smart Materials and Structures, 2016, 25 (11), 10.1088/0964-1726/25/11/115042 . hal-01691082

\section{HAL Id: hal-01691082 \\ https://hal.science/hal-01691082}

Submitted on 22 Mar 2018

HAL is a multi-disciplinary open access archive for the deposit and dissemination of scientific research documents, whether they are published or not. The documents may come from teaching and research institutions in France or abroad, or from public or private research centers.
L'archive ouverte pluridisciplinaire HAL, est destinée au dépôt et à la diffusion de documents scientifiques de niveau recherche, publiés ou non, émanant des établissements d'enseignement et de recherche français ou étrangers, des laboratoires publics ou privés. 


\title{
Multimodal vibration damping of a plate by piezoelectric coupling to its analogous electrical network
}

\author{
B Lossouarn ${ }^{1}$, J-F Deü ${ }^{1}$, M Aucejo ${ }^{1}, \mathrm{~K}$ A Cunefare ${ }^{2}$ \\ 1 Structural Mechanics and Coupled Systems Laboratory, Conservatoire \\ National des Arts et Métiers, 292 Rue Saint-Martin, 75003 Paris, France \\ 2 School of Mechanical Enginering, Georgia Institute of Technology, 771 Ferst \\ Drive NW Atlanta, GA 30332 \\ E-mail: boris.lossouarn@cnam.fr
}

\begin{abstract}
Multimodal damping can be achieved by coupling a mechanical structure to an electrical network exhibiting similar modal properties. Focusing on a plate, a new topology for such an electrical analogue is found from a finite difference approximation of the Kirchhoff-Love theory and the use of the direct electromechanical analogy. Discrete models based on element dynamic stiffness matrices are proposed to simulate square plate unit cells coupled to their electrical analogues through two-dimensional piezoelectric transducers. A setup made of a clamped plate covered with an array of piezoelectric patches is built in order to validate the control strategy and the numerical models. The analogous electrical network is implemented with passive components as inductors, transformers and the inherent capacitance of the piezoelectric patches. The effect of the piezoelectric coupling on the dynamics of the clamped plate is significant as it creates the equivalent of a multimodal tuned mass damping. An adequate tuning of the network then yields a broadband vibration reduction. In the end, the use of an analogous electrical network appears as an efficient solution for the multimodal control of a plate.
\end{abstract}

PACS numbers: 85.50.-n, 46.40.Ff, 84.32.-y, 46.70.De, 85.70.Ge

Keywords: vibration control, multimodal damping, piezoelectricity, electrical analogue, clamped plate, passive network

Submitted to: Smart Mater. Struct. 


\section{Introduction}

In 1991, Hagood and von Flotow [1] showed that the resonant piezoelectric shunt made of an inductor and a resistor is equivalent to a tuned mass damper. About 20 years later, distributed control solutions involving an array of resonant piezoelectric shunts have been proposed in order to damp vibrations of plates $[2,3,4,5]$. One of the main limits is that the required inductance values are usually very large $[6,7]$. It is still possible to generate synthetic inductors with operational amplifiers $[8$, 7] but the system then requires a power supply. Furthermore, the narrow bandwidth of classical resonant shunt in the low-frequency range means that two modes cannot be controlled simultaneously with a single inductor. For this purpose, multiplemode shunt circuits were proposed $[9,8,10]$ but the number of electrical components makes the practical implementation difficult when considering a large array of piezoelectric patches. A broadband control can still be implemented with negative capacitance, which simplifies the electrical layout and offers good performances $[11,12,13]$. The main drawback is that a negative capacitance needs to be synthesized with an active circuit and the control can thus suffer from stability issues.

A solution that generates a passive and broadband control of a plate was proposed by Vidoli and dell'Isola [14]. It consists of an interconnection of piezoelectric patches through distributed impedances, which reduce the inductance requirements [15]. The objective is to build a distributed controller that gives rise to $2 \mathrm{D}$ electrical modes approximating the mechanical modes of interest over the whole area of the structure. The electrical network thus needs to offer resonances at specific target frequencies, as well as a correlation between the electrical and mechanical mode shapes in order to generate an adequate electromechanical coupling. As presented by Batra et al. [16], interconnections with passive branches made of inductors and capacitors allow creating suitable multimodal networks. However, this solution is based on the use of external capacitors, while it has been noted that such additional components decreases the coupling and thus the damping performances $[6,17]$. No external capacitors are yet required when considering the implementation of analogous electrical networks because one can rely on the inherent capacitance of the piezoelectric patches [18]. Then, it has been shown that the energy transfer between the mechanical and electrical systems is maximized when considering an electrical network which offers partial differential equations analogous to those of the structure to control. This means that the electrical analogue represents the optimal passive network to be used for multimodal vibration control $[19,20]$. Therefore, suitable topologies can be found by applying an electromechanical analogy [21]. Around 1950's, MacNeal et al. already proposed and validated electrical analogues for numerous mechanical structures as rods, beams [22, 23], plates [22, 24] and shells [25]. Those passive networks finally reappeared for the analogous control of one-dimensional structures $[18,20]$. With this approach, the number of components per piezoelectric patch is independent of the number of modes that are targeted. For the control of thin plates, Alessandroni et al. [26, 27] introduced an analogous network ensuring a broadband piezoelectric damping. Unfortunately, the large number of electrical components makes difficult its practical implementation. A simpler electrical network can be obtained by reconsidering the Kirchhoff-Love theory. A new topology is presented in this paper together with a modeling of the two-dimensional piezoelectric coupling. The control strategy is then applied to the damping of a clamped plate, effectively implementing a distributed multimodal tuned mass damper. Interested readers can refer to [28] for more details about the robustness of the control strategy.

The present paper is organized as follows. In section 2, the plate electrical analogue is obtained by extending a procedure previously applied to rods and beams $[29,30]$. It consists in a finite difference approximation of the equations describing the mechanical medium, followed by a direct electromechanical analogy. In section 3 , a square piezoelectric laminate is modeled by an equivalent two-dimensional circuit. A discrete modeling of the mechanical and electrical unit cells then allows the definition of a unified dynamic stiffness matrix formulation involving both mechanical and electrical variables. For the purpose of validating the numerical model as well as the damping strategy, an experimental setup is presented in section 4. An array of piezoelectric patches covers a clamped aluminum plate and an analogous network is built with passive electrical components. In section 5, the model 
is validated through experiments that also show the efficiency of the proposed multimodal control. Depending on the application, the electrical network is tuned by adding resistors or by modifying the values of the inductors. Significant vibration reduction is observed, which validates the interest of the damping strategy.

\section{Electrical analogue of a square plate unit cell}

A finite difference method is applied to the KirchhoffLove plate equation of motion in order to get a discrete mechanical model. This model is then converted in the electrical domain by applying a direct electromechanical analogy. Some elements are finally added to the electrical circuit for later modeling of the electrical analogue.

\subsection{Finite difference model of a plate}

The Kirchhoff-Love differential equation for an elastic plate of thickness $h$ and density $\rho$ is given by

$-D\left(\frac{\partial^{4} w}{\partial x^{4}}+2 \frac{\partial^{4} w}{\partial x^{2} \partial y^{2}}+\frac{\partial^{4} w}{\partial y^{4}}\right)=\rho h \frac{\partial^{2} w}{\partial t^{2}}$.

The constant $D=\frac{Y h^{3}}{12\left(1-\nu^{2}\right)}$ represents the bending stiffness, where $Y$ is the Young's modulus of the plate, $\nu$ is its Poisson's ratio. Furthermore, $w=W(x, y) g(t)$ is the transverse displacement, $x$ and $y$ are the two space variables and $t$ is the time variable. Under harmonic motion, $g(t)$ is a trigonometric function which satisfy $\partial^{2} g / \partial t^{2}=-\omega^{2} g$, where $\omega$ is the angular frequency. The state variable formulation of the differential equation (1) can then be expressed as follows:

$$
\begin{aligned}
& \frac{\partial Q_{x}}{\partial x}+\frac{\partial Q_{y}}{\partial y}=-\rho h a \omega^{2} W \\
& Q_{x}=\frac{\partial M_{x}}{\partial x}-\frac{\partial M_{x y}}{\partial y} \\
& Q_{y}=\frac{\partial M_{y}}{\partial y}-\frac{\partial M_{x y}}{\partial x} \\
& M_{x}=-a D\left(\frac{\partial^{2} W}{\partial x^{2}}+\nu \frac{\partial^{2} W}{\partial y^{2}}\right) \\
& M_{y}=-a D\left(\frac{\partial^{2} W}{\partial y^{2}}+\nu \frac{\partial^{2} W}{\partial x^{2}}\right) \\
& M_{x y}=a D(1-\nu) \frac{\partial^{2} W}{\partial x \partial y}
\end{aligned}
$$

This corresponds to the classical variables used by Timoshenko [31] in which we have introduced the constant $a$, that is the side of a square plate unit cell, in order to get variables $Q$ and $M$ homogeneous to forces and moments. The variables $Q_{x}$ and $Q_{y}$ are shear forces, while $M_{x}, M_{y}$ and $M_{x y}$ are respectively the bending moments along $x$, the bending moment along $y$ and the twisting moment. Equation (1) can

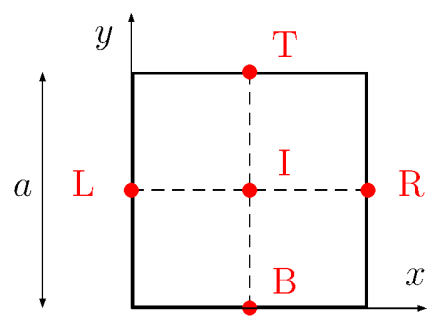

Figure 1: Finite difference grid for a square unit cell.

also be written as

$$
\begin{aligned}
& \frac{\partial Q_{x}}{\partial x}+\frac{\partial Q_{y}}{\partial y}=-\rho h a \omega^{2} W \\
& Q_{x}=-\frac{\partial M}{\partial x} \\
& Q_{y}=-\frac{\partial M}{\partial y} \\
& M=a D\left(\frac{\partial \theta_{x}}{\partial x}+\frac{\partial \theta_{y}}{\partial y}\right) \\
& \theta_{x}=\frac{\partial W}{\partial x} \\
& \theta_{y}=\frac{\partial W}{\partial y}
\end{aligned}
$$

The variables $Q_{x}$ and $Q_{y}$ still represent shear forces and their definition is actually the same as in equation (2). The angles along the principal directions, $\theta_{x}$ and $\theta_{y}$, satisfy the Kirchhoff-Love assumption which states that the segments normal to the undeformed midplane remain straight after deformation. Yet, the moment variable $M$ appearing in equation (3) does not refer to any classical variable of the plate theory [31]. It is here introduced for the definition of an adequate finite difference model.

The continuous plate element is discretized by applying the finite difference scheme

$$
\begin{array}{lll}
\left(\frac{\partial[\cdot]}{\partial x}\right)_{\mathrm{L}}=\frac{[\cdot]_{\mathrm{I}}-[\cdot]_{\mathrm{L}}}{a / 2} & \left(\frac{\partial[\cdot]}{\partial y}\right)_{\mathrm{B}}=\frac{[\cdot]_{\mathrm{I}}-[\cdot]_{\mathrm{B}}}{a / 2} \\
\left(\frac{\partial[\cdot]}{\partial x}\right)_{\mathrm{I}}=\frac{[\cdot]_{\mathrm{R}}-[\cdot]_{\mathrm{L}}}{a} & \text { and } & \left(\frac{\partial[\cdot]}{\partial y}\right)_{\mathrm{I}}=\frac{[\cdot]_{\mathrm{T}}-[\cdot]_{\mathrm{B}}}{a}, \\
\left(\frac{\partial[\cdot]}{\partial x}\right)_{\mathrm{R}}=\frac{[\cdot]_{\mathrm{R}}-[\cdot]_{\mathrm{I}}}{a / 2} & & \left(\frac{\partial[\cdot]}{\partial y}\right)_{\mathrm{T}}=\frac{[\cdot]_{\mathrm{T}}-[\cdot]_{\mathrm{I}}}{a / 2}
\end{array}
$$

where ' $\mathrm{I}$ ' is the position at the center of the square unit cell and ' $L$ ', 'R', 'B' and 'T' refer to the left, right, bottom and top sides, according to the grid in figure 1. The finite difference approximation is applied to equation (3), which gives

$Q_{\mathrm{R}}-Q_{\mathrm{L}}+Q_{\mathrm{T}}-Q_{\mathrm{B}}=-m \omega^{2} W_{\mathrm{I}}$

$Q_{\mathrm{L}}=-\frac{M_{\mathrm{I}}-M_{\mathrm{L}}}{a / 2}$

$Q_{\mathrm{R}}=-\frac{M_{\mathrm{R}}-M_{\mathrm{I}}}{a / 2}$

$Q_{\mathrm{B}}=-\frac{M_{\mathrm{I}}-M_{\mathrm{B}}}{a / 2}$

$Q_{\mathrm{T}}=-\frac{M_{\mathrm{T}}^{a}-M_{\mathrm{I}}}{a / 2}$

$M_{\mathrm{I}}=D\left(\theta_{\mathrm{R}}-\theta_{\mathrm{L}}+\theta_{\mathrm{T}}-\theta_{\mathrm{B}}\right)$

$\theta_{\mathrm{L}}=\frac{W_{\mathrm{I}}-W_{\mathrm{L}}}{a / 2}$

$\theta_{\mathrm{R}}=\frac{W_{\mathrm{R}}-W_{\mathrm{I}}}{a / 2}$

$\theta_{\mathrm{B}}=\frac{W_{\mathrm{I}}-W_{\mathrm{B}}}{a / 2}$

$\theta_{\mathrm{T}}=\frac{W_{\mathrm{T}}-W_{\mathrm{I}}}{a / 2}$

where $m=\rho h a^{2}$ is the mass of the square plate unit cell. 


\subsection{Analogous electrical circuit}

The finite difference model in equation (5) can be converted into its equivalent circuit model made of discrete electrical components. Previous studies on plate electrical analogues $[27,22]$ were based on the indirect or force-current electromechanical analogy. Here, we rather focus on the direct electromechanical analogy, which is compatible with the passive representation of a piezoelectric transducer $[21,32]$. With this analogy, electromechanical problems involving piezoelectric coupling can be modeled with passive electrical networks $[29,30]$. The direct analogy is based on the velocity-current and force-voltage equivalences. Then, considering that the dot notation refer to the time derivative and that ' $\mathrm{j}$ ' is the imaginary unit, equation (5) can be written as

$$
\begin{aligned}
& -Q_{\mathrm{L}}-\left(Q_{\mathrm{B}}-Q_{\mathrm{T}}\right)+Q_{\mathrm{R}}=\mathrm{j} \omega m \dot{W}_{\mathrm{I}} \\
& -M_{\mathrm{L}}+M_{\mathrm{I}}=-\frac{a}{2} Q_{\mathrm{L}} \\
& -M_{\mathrm{I}}+M_{\mathrm{R}}=-\frac{a}{2} Q_{\mathrm{R}} \\
& -M_{\mathrm{B}}+M_{\mathrm{I}}=-\frac{a}{2} Q_{\mathrm{B}} \\
& -M_{\mathrm{I}}+M_{\mathrm{T}}=-\frac{a}{2} Q_{\mathrm{T}} \\
& -M_{\mathrm{I}}=D \\
& \frac{\mathrm{j} \omega}{2}\left(\dot{\theta}_{\mathrm{L}}-\dot{\theta}_{\mathrm{R}}+\dot{\theta}_{\mathrm{B}}-\dot{\theta}_{\mathrm{T}}\right) \\
& \frac{a}{2} \dot{\theta}_{\mathrm{L}}=\dot{W}_{\mathrm{I}}-\dot{W}_{\mathrm{L}} \\
& \frac{a}{2} \dot{\theta}_{\mathrm{R}}=\dot{W}_{\mathrm{R}}-\dot{W}_{\mathrm{I}} \\
& \frac{a}{2} \dot{\theta}_{\mathrm{B}}=\dot{W}_{\mathrm{I}}-\dot{W}_{\mathrm{B}} \\
& \frac{a}{2} \dot{\theta}_{\mathrm{T}}=\dot{W}_{\mathrm{T}}-\dot{W}_{\mathrm{I}}
\end{aligned}
$$

which corresponds to the constitutive equations of the electrical circuit in figure 2 . The inductance represents the mass $m$ of the unit cell and the capacitance is the inverse of the bending stiffness $D$, in agreement with the direct electromechanical analogy [21, 33]. Furthermore, the electrical transformers generate the forward and backward finite differences that satisfy the definition of $\theta$ and $Q$ as spatial derivatives of $w$ and $-M$, respectively. The proposed unit cell can finally be seen as a two-dimensional extension of the beam electrical analogue $[20,30]$. The electrical circuit in figure 2 has to be replicated along the $x$ and $y$ directions in order to create the electrical analogue of a plate. We obtain a network with fewer electrical components than the topology introduced by Alessandroni et al. [26, 27]. As a consequence, the proposed electrical analogue simplifies the practical implementation.

\subsection{Modified electrical unit cell}

When considering the implementation of a direct electrical analogue for a piezoelectric damping purpose [30], it is actually not required to build electrical unit cells with and inductance $L=m$, a capacitance $C=1 / D$ and a transformer ratio $\hat{a}=a$. Indeed, we only need to ensure that the modal properties of the electrical network are identical to those of the considered discretized mechanical structure. By considering a discrete formulation of equation (1), it is found that two finite difference models based on different plate dimensions and material properties would still lead to an identical eigenvalue problem if they offer the same ratio $D /\left(a^{4} \rho h\right)$. As $m=\rho h a^{2}$, when focusing on an analogous network the previous condition becomes

$\frac{1}{\hat{a}^{2}} \frac{1}{L C}=\frac{1}{a^{2}} \frac{D}{m}$.

This equation thus gives a solution for subsequent tuning of the electrical network, which has to approximate the modal properties of the structure to control in order to optimize the piezoelectric coupling [15].

The unit cell of the analogous network is represented in figure 3 with the electrical variables that are going to be used in the following electromechanical models. Here, we take into account the inherent damping present in passive electrical components. The damping in the inductor can be modeled by a series resistance $R_{\mathrm{S}}^{L}$ and a parallel resistance $R_{\mathrm{p}}^{L}$. Resistors $R_{\mathrm{S}}^{T} / 2$ and $R_{\mathrm{S}}^{T^{\star}}$ are also introduced to model the joule effect in the windings of the transformers, which is usually non negligible. A last damping element to consider is the eventual resistance $R_{\mathrm{S}}^{+}$that can be added in series with the inductors $L$ for a tuning purpose. In the end, note the addition of capacitors $C_{0} / 2$ in the electrical circuit. Those are represented to allow the definition of a "dynamic stiffness matrix" in the following section. Indeed, equation (5) shows that the displacements are not independent variables. If all four angles have prescribed values, the choice of a single displacement enforces the value of the other ones. In order to relax these kinematic constraints linking angles and displacements, virtual degrees of freedom have to be added through capacitors in both electrical circuits representing the mechanical and electrical unit cells. Therefore, the capacitance $C_{0}$ in figure 3 is only a numerical parameter. It has to be small compared to $C$ but high enough to avoid numerical issues. A value of $C_{0}$ equal to $C \times 10^{-6}$ is appropriate for the following computations.

\section{Modeling of the coupled problem}

The 3D linear theory of piezoelectricity leads to a global model describing a square piezoelectric laminate. From this model, it is drawn an electrical circuit representing the two-dimensional piezoelectric coupling. This new building block allows coupling the finite difference model of a plate unit cell to its analogous electrical network. A discrete electromechanical unit cell is thus defined and it is then refined under a more accurate macro unit cell. 


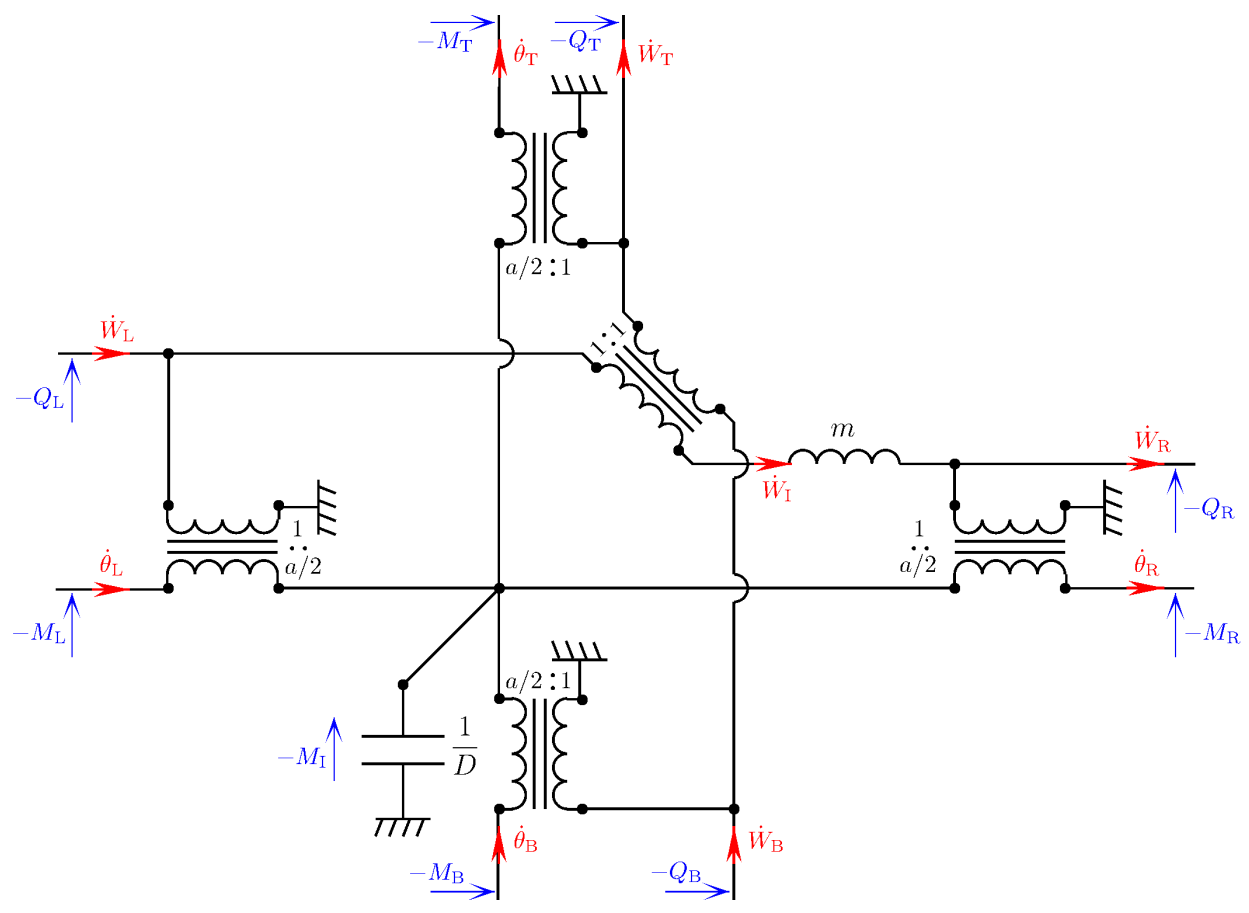

Figure 2: Electrical unit cell as a direct analogue of the plate finite difference model.

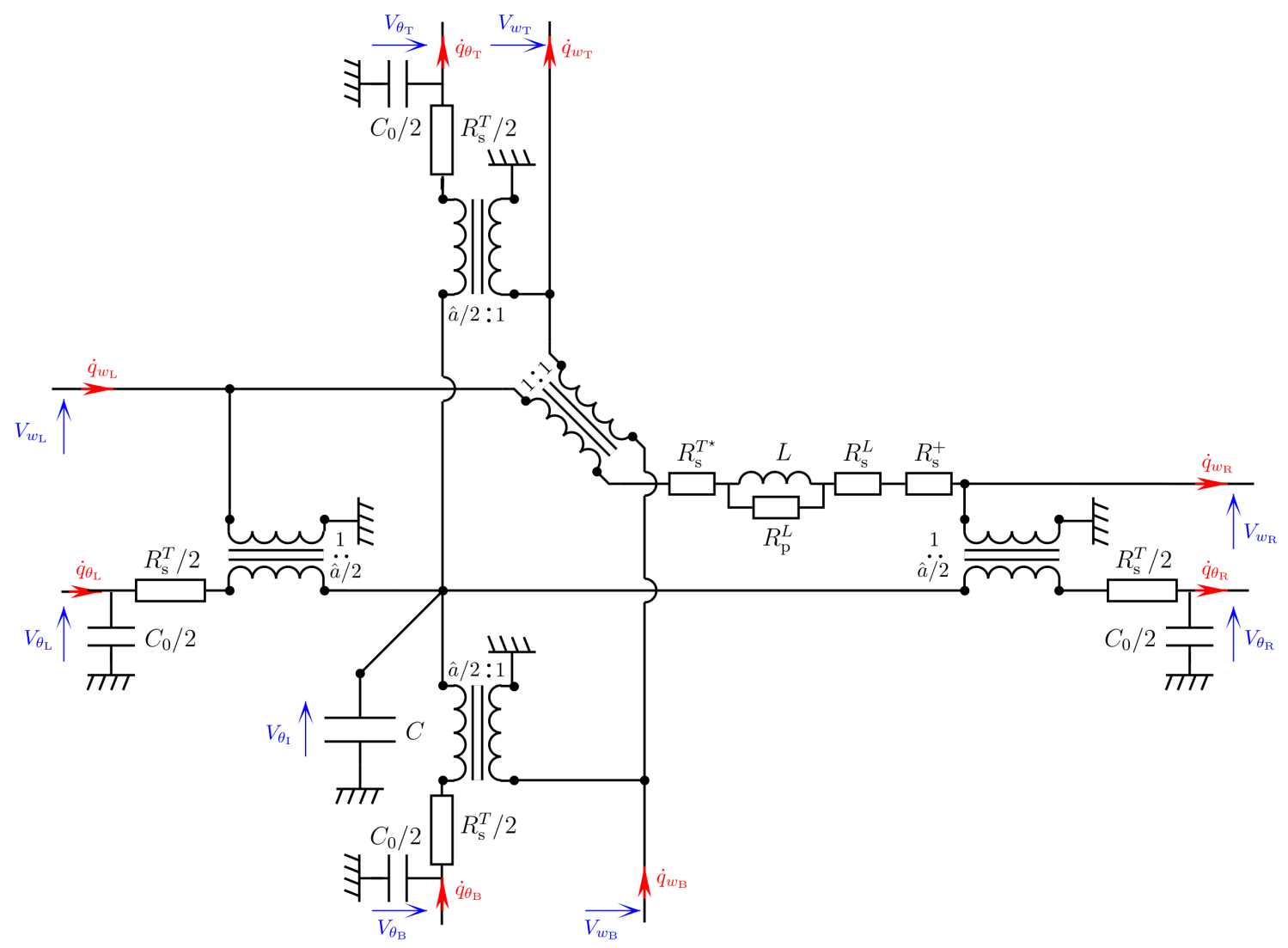

Figure 3: Modified unit cell for the calculation of an element dynamic stiffness matrix. 


\subsection{Square piezoelectric plate}

According to the IEEE Standards on piezoelectricity [34], the 3D linear theory for a transverse isotropic PZT ceramic polarized in the direction '3' can be expressed as

$\left[\begin{array}{c}\varepsilon_{1} \\ \varepsilon_{2} \\ \varepsilon_{3} \\ \varepsilon_{4} \\ \varepsilon_{5} \\ \varepsilon_{6} \\ D_{1} \\ D_{2} \\ D_{3}\end{array}\right]=\left[\begin{array}{cccccc|ccc}s_{11}^{E} & s_{12}^{E} & s_{13}^{E} & 0 & 0 & 0 & 0 & 0 & d_{31} \\ s_{12}^{E} & s_{11}^{E} & s_{13}^{E} & 0 & 0 & 0 & 0 & 0 & d_{31} \\ s_{13}^{E} & s_{13}^{E} & s_{33}^{E} & 0 & 0 & 0 & 0 & 0 & d_{33} \\ 0 & 0 & 0 & s_{44}^{E} & 0 & 0 & 0 & d_{15} & 0 \\ 0 & 0 & 0 & 0 & s_{44}^{E} & 0 & d_{15} & 0 & 0 \\ 0 & 0 & 0 & 0 & 0 & s_{66}^{E} & 0 & 0 & 0 \\ \hline 0 & 0 & 0 & 0 & d_{15} & 0 & \epsilon_{11}^{\sigma} & 0 & 0 \\ 0 & 0 & 0 & d_{15} & 0 & 0 & 0 & \epsilon_{11}^{\sigma} & 0 \\ d_{31} & d_{31} & d_{33} & 0 & 0 & 0 & 0 & 0 & \epsilon_{33}^{\sigma}\end{array}\right]\left[\begin{array}{c}\sigma_{1} \\ \sigma_{2} \\ \sigma_{3} \\ \sigma_{4} \\ \sigma_{5} \\ \sigma_{6} \\ E_{1} \\ E_{2} \\ E_{3}\end{array}\right]$

where $\varepsilon_{i}, \sigma_{i}, D_{i}$ and $E_{i}$ represent respectively the strain, the stress, the electric displacement and the electric field along the three space directions $(i=$ $\{1,2,3\})$. The constants $s_{i j}^{E}$ refer to the elastic compliance at constant electric field, $d_{i j}$ represents the piezoelectric constants and $\epsilon_{i j}^{\sigma}$ is the permittivity at constant stress. Regarding a thin piezoelectric plate whose thickness corresponds to the direction of polarization, a classical hypothesis is to consider that the electric field is equal to zero along the principal directions [35], i.e. $E_{1}=E_{2}=0$. The plane stress hypothesis $\sigma_{3}=0$ then leads to the reduced matrix

$\left[\begin{array}{c}\varepsilon_{1} \\ \varepsilon_{2} \\ \varepsilon_{4} \\ \varepsilon_{5} \\ \varepsilon_{6} \\ D_{3}\end{array}\right]=\left[\begin{array}{cccccc}s_{11}^{E} & s_{12}^{E} & 0 & 0 & 0 & d_{31} \\ s_{12}^{E} & s_{11}^{E} & 0 & 0 & 0 & d_{31} \\ 0 & 0 & s_{44}^{E} & 0 & 0 & 0 \\ 0 & 0 & 0 & s_{44}^{E} & 0 & 0 \\ 0 & 0 & 0 & 0 & s_{66}^{E} & 0 \\ d_{31} & d_{31} & 0 & 0 & 0 & \epsilon_{33}^{\sigma}\end{array}\right]\left[\begin{array}{c}\sigma_{1} \\ \sigma_{2} \\ \sigma_{4} \\ \sigma_{5} \\ \sigma_{6} \\ E_{3}\end{array}\right]$.

Consequently, it is found that

$$
\begin{aligned}
& \varepsilon_{1}+\varepsilon_{2}=\left(s_{11}^{E}+s_{12}^{E}\right)\left(\sigma_{1}+\sigma_{2}\right)+2 d_{31} E_{3} \\
& D_{3}=d_{31}\left(\sigma_{1}+\sigma_{2}\right)+\epsilon_{33}^{\sigma} E_{3}
\end{aligned} .
$$

From the definition of the material constants $s_{11}^{E}$ and $s_{12}^{E}[34,35]$, we get $s_{11}^{E}+s_{12}^{E}=\left(1-\nu_{\mathrm{p}}\right) / Y_{\mathrm{p}}^{E}$, where $\nu_{\mathrm{p}}$ is the Poisson's ratio of the piezoelectric material and $Y_{\mathrm{p}}^{E}=1 / s_{11}^{E}$ is its Young's modulus when the transverse electric field is equal to zero $\left(E_{3}=0\right)$. Equation (10) can then be reorganized into

$$
\begin{aligned}
& \sigma_{1}+\sigma_{2}=\frac{Y_{\mathrm{p}}^{E}}{1-\nu_{\mathrm{p}}}\left(\varepsilon_{1}+\varepsilon_{2}\right)-d_{31} \frac{2 Y_{\mathrm{p}}^{E}}{1-\nu_{\mathrm{p}}} E_{3} \\
& D_{3}=d_{31} \frac{Y_{\mathrm{p}}^{E}}{1-\nu_{\mathrm{p}}}\left(\varepsilon_{1}+\varepsilon_{2}\right)+\epsilon_{33}^{\varepsilon^{\star}} E_{3}
\end{aligned},
$$

where

$\epsilon_{33}^{\varepsilon^{\star}}=\epsilon_{33}^{\sigma}\left(1-\frac{2}{1-\nu_{\mathrm{p}}} \frac{d_{31}^{2}}{s_{11}^{E} \epsilon_{33}^{\sigma}}\right)$

is the equivalent permittivity of a piezoelectric plate which is blocked along its principal directions, i.e. $\varepsilon_{1}=$ $\varepsilon_{2}=0$.
For a plate subjected to bending motion, the strains $\varepsilon_{1}$ and $\varepsilon_{2}$ can be expressed as

$$
\varepsilon_{1}=-z \frac{\partial \theta_{x}}{\partial x} \quad \text { and } \quad \varepsilon_{2}=-z \frac{\partial \theta_{y}}{\partial y},
$$

where $x$ and $y$ refer to the principal directions ' 1 ' and ' 2 ', and $z$ refers to the transverse direction ' 3 '. Considering the plate theory [31] for a square unit cell of side $l_{\mathrm{p}}$, the bending moments $M_{x}$ and $M_{y}$ that have been defined in equation (2) are related to the stresses $\sigma_{1}$ and $\sigma_{2}$ through

$$
M_{x}=l_{\mathrm{p}} \int_{z} \sigma_{1} z d z \quad \text { and } \quad M_{y}=l_{\mathrm{p}} \int_{z} \sigma_{2} z d z .
$$

Furthermore, the total electric charge $q_{\mathrm{p}}$ and the voltage $V_{\mathrm{p}}$ between the two electrodes of the piezoelectric element are defined as

$$
q_{\mathrm{p}}=-\int_{x} \int_{y} D_{3} d x d y \quad \text { and } \quad E_{3}=-\frac{V_{\mathrm{p}}}{h_{\mathrm{p}}},
$$

where $h_{\mathrm{p}}$ is the thickness of the piezoelectric plate [7, 36]. As we consider a thin piezoelectric plate compared to its distance $\lambda$ from the mid-surface, i.e. $\lambda \gg h_{\mathrm{p}}$ so that $\int_{\lambda-h_{\mathrm{p}} / 2}^{\lambda+h_{\mathrm{p}} / 2} z d z / h_{\mathrm{p}} \approx \lambda$, then equation (11) gives

$$
\begin{aligned}
& M_{x}+M_{y}=-\frac{Y_{\mathrm{p}}^{E} I_{\mathrm{p}}}{1-\nu_{\mathrm{p}}}\left(\frac{\partial \theta_{x}}{\partial x}+\frac{\partial \theta_{y}}{\partial y}\right)+\lambda l_{\mathrm{p}} d_{31} \frac{2 Y_{\mathrm{p}}^{E}}{1-\nu_{\mathrm{p}}} V_{\mathrm{p}} \\
& q_{\mathrm{p}}=\lambda l_{\mathrm{p}} d_{31} \frac{Y_{\mathrm{p}}^{E}}{1-\nu_{\mathrm{p}}}\left(\overline{\Delta \theta_{x}}+\overline{\Delta \theta}_{y}\right)+\epsilon_{33}^{\varepsilon^{\star}} l_{\mathrm{p}}^{2} V_{\mathrm{p}}
\end{aligned}
$$

where $\overline{\Delta \theta}_{x}=\frac{1}{l_{\mathrm{p}}} \int_{0}^{l_{\mathrm{p}}} \Delta \theta_{x}(y) d y$ and $\overline{\Delta \theta}_{y}=$ $\frac{1}{l_{\mathrm{p}}} \int_{0}^{l_{\mathrm{p}}} \Delta \theta_{y}(x) d x$ represent the average differences of the angles at opposite sides, and $I_{\mathrm{p}}=l_{\mathrm{p}} \int_{\lambda-h_{\mathrm{p}} / 2}^{\lambda+h_{\mathrm{p}} / 2} z^{2} d z$ is the second moment of area. Recall that the sum of the bending moment is related to the variable $M$ defined in equation (3) through

$M_{\mathrm{p}}=-\frac{1}{1+\nu}\left(M_{x}+M_{y}\right)$.

So, equation (16) is equivalent to

$$
\begin{aligned}
& M_{\mathrm{p}}=l_{\mathrm{p}} D_{\mathrm{p}}^{E}\left(\frac{\partial \theta_{x}}{\partial x}+\frac{\partial \theta_{y}}{\partial y}\right)-\frac{2 e_{\mathrm{p}}}{1-\nu_{\mathrm{p}}^{2}} V_{\mathrm{p}} \\
& q_{\mathrm{p}}=\frac{e_{\mathrm{p}}}{1-\nu_{\mathrm{p}}}\left(\overline{\Delta \theta}_{x}+\overline{\Delta \theta}_{y}\right)+C_{\mathrm{p}}^{\varepsilon} V_{\mathrm{p}}
\end{aligned},
$$

where $D_{\mathrm{p}}^{E}=\frac{Y_{\mathrm{p}}^{E} I_{\mathrm{p}}}{\left(1-\nu_{\mathrm{p}}^{2}\right) l_{\mathrm{p}}}$ is the short-circuited bending stiffness, $C_{\mathrm{p}}^{\varepsilon}=\epsilon_{33}^{\varepsilon^{\star}} \frac{l_{\mathrm{p}}^{2}}{h_{\mathrm{p}}}$ is the blocked piezoelectric capacitance and $e_{\mathrm{p}}=\lambda l_{\mathrm{p}} d_{31} Y_{\mathrm{p}}^{E}$ is the coupling coefficient. It is noticed that equation (18) does not offer a symmetrical reciprocity [21], i.e. the global coefficients related to the piezoelectric coupling are not the same for the mechanical and electrical contributions. To model the coupling with a passive circuit involving an electrical transformer [32, 29, 30], the symmetry condition is yet required. Equation (18) is thus written as

$$
\begin{aligned}
& M_{\mathrm{p}}=l_{\mathrm{p}} D_{\mathrm{p}}^{E}\left(\frac{\partial \theta_{x}}{\partial x}+\frac{\partial \theta_{y}}{\partial y}\right)-\sqrt{2\left(1+\nu_{\mathrm{p}}\right)} \frac{e_{\mathrm{p}}}{1-\nu_{\mathrm{p}}^{2}}\left[\sqrt{\frac{2}{1+\nu_{\mathrm{p}}}} V_{\mathrm{p}}\right] \\
& {\left[\sqrt{\frac{2}{1+\nu_{\mathrm{p}}}} q_{\mathrm{p}}\right]=\sqrt{2\left(1+\nu_{\mathrm{p}}\right)} \frac{e_{\mathrm{p}}}{1-\nu_{\mathrm{p}}^{2}}\left(\overline{\Delta \theta}_{x}+\overline{\Delta \theta}_{y}\right)+C_{\mathrm{p}}^{\varepsilon}\left[\sqrt{\frac{2}{1+\nu_{\mathrm{p}}}} V_{\mathrm{p}}\right]}
\end{aligned}
$$



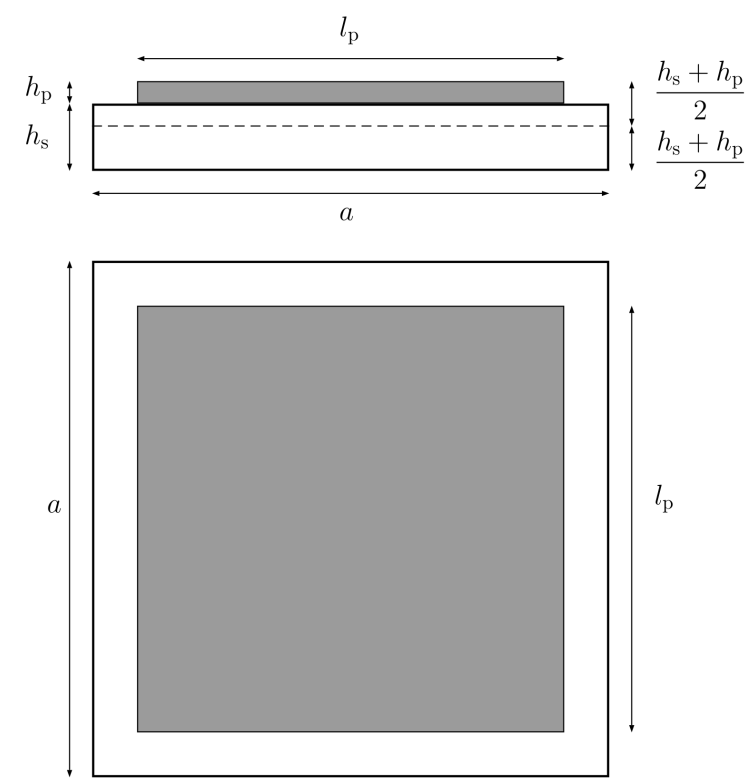

Figure 4: Square piezoelectric laminate with a single piezoelectric patch.

which is equivalent to

$$
\begin{aligned}
& M_{\mathrm{p}}=l_{\mathrm{p}} D_{\mathrm{p}}^{E}\left(\frac{\partial \theta_{x}}{\partial x}+\frac{\partial \theta_{y}}{\partial y}\right)-e_{\mathrm{p}}^{\star} V_{\mathrm{p}}^{\star} \\
& q_{\mathrm{p}}^{\star}=e_{\mathrm{p}}^{\star}\left(\overline{\Delta \theta}_{x}+\overline{\Delta \theta}_{y}\right)+C_{\mathrm{p}}^{\varepsilon} V_{\mathrm{p}}^{\star}
\end{aligned},
$$

where $e_{\mathrm{p}}^{\star}=\frac{\sqrt{2\left(1+\nu_{\mathrm{p}}\right)}}{1-\nu_{\mathrm{p}}^{2}} e_{\mathrm{p}}, q_{\mathrm{p}}^{\star}=\sqrt{\frac{2}{1+\nu_{\mathrm{p}}}} q_{\mathrm{p}}$ and $V_{\mathrm{p}}^{\star}=$ $\sqrt{\frac{2}{1+\nu_{\mathrm{p}}}} V_{\mathrm{p}}$. Finally, equation (20) is based on a symmetrical coupling formulation that can be extended to the case of a square unit cell involving a piezoelectric patch covering an elastic substrate.

\subsection{Global model for a square piezoelectric laminate}

A piezoelectric laminate is made of a superposition of piezoelectric and purely elastic layers [36]. A simple laminate is represented in figure 4 , where a square piezoelectric patch of side $l_{\mathrm{p}}$ and thickness $h_{\mathrm{p}}$ covers an elastic structure of side $a$ and thickness $h_{\mathrm{s}}$. On a global scale, if we consider that the piezoelectric laminate can be approximated by a homogeneous piezoelectric medium, a similar form as in equation (20) can be used to describe the piezoelectric coupling in the whole laminate:

$$
\begin{aligned}
& M=a D^{E}\left(\frac{\partial \theta_{x}}{\partial x}+\frac{\partial \theta_{y}}{\partial y}\right)-e_{\theta} V_{\theta_{\mathrm{I}}} . \\
& q_{\theta_{\mathrm{I}}}=e_{\theta}\left(\overline{\Delta \theta}_{x}+\overline{\Delta \theta}_{y}\right)+C^{\varepsilon} V_{\theta_{\mathrm{I}}}
\end{aligned}
$$

If $\nu_{\mathrm{s}}$ is the Poisson's ratio of the elastic structure, the global bending stiffness of the laminate $D^{E}$ is defined from

$$
\frac{1}{D^{E}}=\frac{l_{\mathrm{p}}}{\frac{Y_{\mathrm{s}}}{1-\nu_{\mathrm{s}}^{2}} I_{\mathrm{s}}+\frac{Y_{\mathrm{p}}^{E}}{1-\nu_{\mathrm{p}}^{2}} I_{\mathrm{p}}}+\frac{a-l_{\mathrm{p}}}{\frac{Y_{\mathrm{s}}}{1-\nu_{\mathrm{s}}^{2}} I_{\mathrm{s}}},
$$

which corresponds to a series of two bending stiffness elements, one for the segment with piezoelectric material and one for the segments without piezoelectric material. $I_{\mathrm{s}}$ and $I_{\mathrm{p}}$ are the second moments of area for the elastic and piezoelectric plates. When the midsurface is at a constant position $\left(h_{\mathrm{s}}+h_{\mathrm{p}}\right) / 2$ from the free surface of the piezoelectric patch, as represented in figure 4 , the second moments of area are

$$
\begin{aligned}
& I_{\mathrm{p}}=l_{\mathrm{p}} \int_{\frac{h_{\mathrm{s}}-h_{\mathrm{p}}}{2}}^{\frac{h_{\mathrm{s}}+h_{\mathrm{p}}}{2}} z^{2} d z=l_{\mathrm{p}} \frac{\left(h_{\mathrm{s}}+h_{\mathrm{p}}\right)^{3}-\left(h_{\mathrm{s}}-h_{\mathrm{p}}\right)^{3}}{24} \\
& I_{\mathrm{s}}=a \int_{-\frac{h_{\mathrm{s}}+h_{\mathrm{p}}}{2}}^{\frac{h_{\mathrm{s}}-h_{\mathrm{p}}}{2}} z^{2} d z=a \frac{\left(h_{\mathrm{s}}-h_{\mathrm{p}}\right)^{3}+\left(h_{\mathrm{s}}+h_{\mathrm{p}}\right)^{3}}{24}
\end{aligned}
$$

This expression is valid only if the Young's moduli of both material have close values and if the piezoelectric plate covers most of the elastic surface $\left(l_{\mathrm{p}} \approx a\right)$, which is verified in the following application. If this is not the case, a position of the mid-surface different from $\left(h_{\mathrm{s}}+h_{\mathrm{p}}\right) / 2$ would lead to another expression for the second moments of area.

The global coupling coefficient $e_{\theta}$ in equation (21) could be eventually obtained from $3 \mathrm{D}$ calculations [37] but it has been remarked that the bonding layer between the main structure and the patches has usually a non-negligible influence. A more direct method to get the actual value for $e_{\theta}$ is to compare the short- and open-circuited responses of the whole piezoelectric structure. A lack of precise modeling of the bonding layer leads us to use the latter approach in the following comparisons with experimental results. The same conclusions arise for the determination of the blocked capacitance $C^{\varepsilon}$ which would require a precise 3D modeling. This can be avoided by direct measurement of the piezoelectric capacitance when blocking transverse displacement of the mechanical structure.

\subsection{Discrete electromechanical unit cell}

The square piezoelectric laminate in figure 4 defines the unit cell of a system based on a periodic array of piezoelectric patches covering an elastic plate. When considering large wavelength compared to the length $a$ of a unit cell,

$$
\begin{aligned}
& \frac{\partial \theta_{x}}{\partial x} \approx \frac{\theta_{\mathrm{R}}-\theta_{\mathrm{L}}}{a}, \quad, \quad \frac{\partial \theta_{y}}{\partial y} \approx \frac{\theta_{\mathrm{T}}-\theta_{\mathrm{B}}}{a} \\
& \Delta \theta_{x} \approx \theta_{\mathrm{R}}-\theta_{\mathrm{L}}, \quad, \quad \bar{\Delta}_{y} \approx \theta_{\mathrm{T}}-\theta_{\mathrm{B}}
\end{aligned},
$$

where $\theta_{\mathrm{R}}, \theta_{\mathrm{L}}, \theta_{\mathrm{T}}$ and $\theta_{\mathrm{B}}$ are the angles on the 4 sides of the unit cell (right, left, top and bottom sides respectively). So, equation (21) is written as

$$
\begin{aligned}
& M_{\mathrm{I}}=D^{E}\left(\theta_{\mathrm{R}}-\theta_{\mathrm{L}}+\theta_{\mathrm{T}}-\theta_{\mathrm{B}}\right)-e_{\theta} V_{\theta_{\mathrm{I}}} \\
& q_{\theta_{\mathrm{I}}}=e_{\theta}\left(\theta_{\mathrm{R}}-\theta_{\mathrm{L}}+\theta_{\mathrm{T}}-\theta_{\mathrm{B}}\right)+C^{\varepsilon} V_{\theta_{\mathrm{I}}}
\end{aligned} .
$$




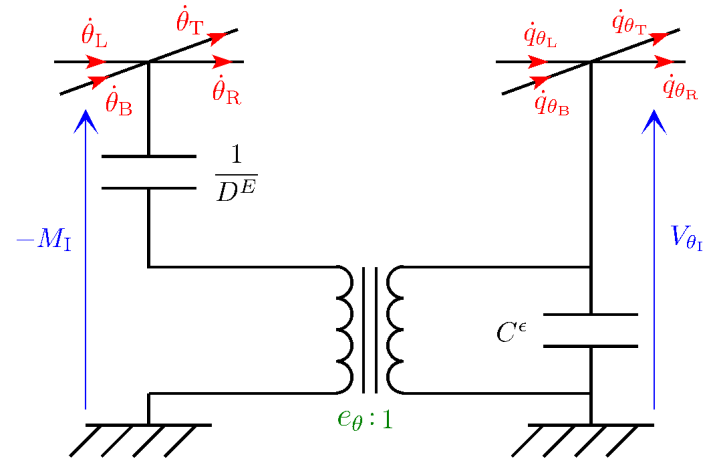

Figure 5: Circuit model of the piezoelectric coupling for a square piezoelectric laminate.

Under harmonic regime, $\dot{\theta}=\mathrm{j} \omega \theta$ on any side of the unit cell and $\dot{q}_{\theta_{\mathrm{I}}}=\mathrm{j} \omega q_{\theta_{\mathrm{I}}}$ so that

$$
\begin{aligned}
& -M_{\mathrm{I}}=\frac{D^{E}}{\mathrm{j} \omega}\left(\dot{\theta}_{\mathrm{L}}-\dot{\theta}_{\mathrm{R}}+\dot{\theta}_{\mathrm{B}}-\dot{\theta}_{\mathrm{T}}\right)+e_{\theta} V_{\theta_{\mathrm{I}}} \\
& V_{\theta_{\mathrm{I}}}=\frac{1}{\mathrm{j} \omega C^{\varepsilon}}\left[\dot{\theta}_{\theta_{\mathrm{I}}}+e_{\theta}\left(\dot{\theta}_{\mathrm{L}}-\dot{\theta}_{\mathrm{R}}+\dot{\theta}_{\mathrm{B}}-\dot{\theta}_{\mathrm{T}}\right)\right]
\end{aligned}
$$

Note that $M_{\mathrm{I}}$ has now the same form as in equation (6) when the piezoelectric coupling coefficient $e_{\theta}$ is equal to zero. With $\dot{q}_{\theta_{\mathrm{I}}}=\dot{q}_{\theta_{\mathrm{L}}}-\dot{q}_{\theta_{\mathrm{R}}}+\dot{q}_{\theta_{\mathrm{B}}}-\dot{q}_{\theta_{\mathrm{T}}}$, equation (26) can be represented by the electrical circuit in figure 5 . This corresponds to a two-dimensional extension of the model used to represent a piezoelectric unit cell subjected to one-dimensional bending motion [30, 32].

The proposed circuit that models the piezoelectric effect along two directions offers a way to couple a square plate unit cell to it analogous electrical network. Indeed, the circuit in figure 5 can be inserted between the unit cell involving mechanical variables in figure 2 and its electrical counterpart in figure 3 . The shortcircuited bending stiffness $D^{E}$ thus replaces the purely mechanical bending stiffness $D$ involved in section 2 and the blocked capacitance $C^{\varepsilon}$ is used instead of $C$. In the end, we get a discrete electromechanical unit cell represented by an electrical circuit involving four external ports per side, i.e. 16 ports in total. The whole set of discrete equations defining the electromechanical unit cell is written down, the internal variables are then eliminated and the system of equations is solved for the side force and voltage variables as linear combinations of the displacement and electric charge variables. We obtain a $16 \times 16$ element "dynamic stiffness matrix" of the coupled system under the form

$\boldsymbol{F}_{c}^{\mathrm{e}}=\boldsymbol{D}_{c}^{\mathrm{e}} \boldsymbol{q}_{c}^{\mathrm{e}}$.

The considered "force" and "displacement" vectors are

$$
\begin{aligned}
& \boldsymbol{F}_{\boldsymbol{c}}^{\mathrm{e}}=\left[\begin{array}{llll}
\boldsymbol{F}_{\mathrm{B}}^{\mathrm{e}} & \boldsymbol{F}_{\mathrm{L}}^{\mathrm{e}} & \boldsymbol{F}_{\mathrm{R}}^{\mathrm{e}} & \boldsymbol{F}_{\mathrm{T}}^{\mathrm{e}}
\end{array}\right]^{T}, \\
& \boldsymbol{q}_{\boldsymbol{c}}^{\mathrm{e}}=\left[\begin{array}{llll}
\boldsymbol{q}_{\mathrm{B}}^{\mathrm{e}} & \boldsymbol{q}_{\mathrm{L}}^{\mathrm{e}} & \boldsymbol{q}_{\mathrm{R}}^{\mathrm{e}} & \boldsymbol{q}_{\mathrm{T}}^{\mathrm{e}}
\end{array}\right]^{T},
\end{aligned}
$$

where

$$
\begin{aligned}
& \boldsymbol{F}_{\mathrm{B}}^{\mathrm{e}}=\left[\begin{array}{llll}
-Q_{\mathrm{B}} & -M_{\mathrm{B}} & V_{w_{\mathrm{B}}} & V_{\theta_{\mathrm{B}}}
\end{array}\right]^{T} \\
& \boldsymbol{F}_{\mathrm{L}}^{\mathrm{e}}=\left[\begin{array}{llll}
-Q_{\mathrm{L}} & -M_{\mathrm{L}} & V_{w_{\mathrm{L}}} & V_{\theta_{\mathrm{L}}}
\end{array}\right]^{T} \\
& \boldsymbol{F}_{\mathrm{R}}^{\mathrm{e}}=\left[\begin{array}{llll}
Q_{\mathrm{R}} & M_{\mathrm{R}} & -V_{w_{\mathrm{R}}} & -V_{\theta_{\mathrm{R}}}
\end{array}\right]^{T} \\
& \boldsymbol{F}_{\mathrm{T}}^{\mathrm{e}}=\left[\begin{array}{llll}
Q_{\mathrm{T}} & M_{\mathrm{T}} & -V_{w_{\mathrm{T}}} & -V_{\theta_{\mathrm{T}}}
\end{array}\right]^{T} \\
& \boldsymbol{q}_{\mathrm{B}}^{\mathrm{e}}=\left[\begin{array}{llll}
W_{\mathrm{B}} & \theta_{\mathrm{B}} & q_{w_{\mathrm{B}}} & q_{\theta_{\mathrm{B}}}
\end{array}\right]_{T}^{T} \\
& \boldsymbol{q}_{\mathrm{L}}^{\mathrm{e}}=\left[\begin{array}{llll}
W_{\mathrm{L}} & \theta_{\mathrm{L}} & q_{w_{\mathrm{L}}} & q_{\theta_{\mathrm{L}}}
\end{array}\right]^{T} \\
& \boldsymbol{q}_{\mathrm{R}}^{\mathrm{e}}=\left[\begin{array}{llll}
W_{\mathrm{R}} & \theta_{\mathrm{R}} & q_{w_{\mathrm{R}}} & q_{\theta_{\mathrm{R}}}
\end{array}\right]_{T}^{T} \\
& \boldsymbol{q}_{\mathrm{T}}^{\mathrm{e}}=\left[\begin{array}{llll}
W_{\mathrm{T}} & \theta_{\mathrm{T}} & q_{w_{\mathrm{T}}} & q_{\theta_{\mathrm{T}}}
\end{array}\right]^{T}
\end{aligned}
$$

The signs in the force vectors are chosen in order to involve external forces applied to the unit cell. As, $Q_{\mathrm{B}}, M_{\mathrm{B}}, Q_{\mathrm{L}}$ and $M_{\mathrm{L}}$ represent mechanical actions applied by the considered unit cell to its bottom or left neighbors, their opposite values are introduced into the force vector. Furthermore, the signs of the electrical variables are the opposites of the signs of the mechanical variables because the voltages are analogous to the opposite of the force contributions, as shown in figure 2. The closed-form expression of $\boldsymbol{D}_{\boldsymbol{c}}^{\mathrm{e}}$ is not given explicitly because the high number of symbolic equations results in a large symbolic matrix which cannot be written compactly. In any case, from a classical assembly of element matrices, the numerical model in equation (27) allows the simulation of a discretized plate coupled to its analogous electrical network.

\subsection{Macro unit cell}

A discrete model based on a large wavelength assumption is not reliable when the considered wavelength approaches the length of the unit cell. In order to maintain a large wavelength condition, one could propose to increase the number of unit cell. However, when considering a practical system, the number of unit cells is usually defined from the choice of the piezoelectric array. A solution is to define a macro unit cell where the mechanical part is refined but the number of unit cells for the electrical network is kept constant. This solution is represented in figure 6(a) where the unit cell in figure 4 is divided in four identical sub-cells. The four piezoelectric patches are connected in parallel and the ratio of piezoelectric material is not modified. This means that the global constants $D^{E}, C^{\varepsilon}$ and $e_{\theta}$ appearing in equation (25) are still the same for the macro unit cell. Also, from the definition of the bending stiffness, the constants $D^{E}$ are equal for the macro unit cell and for the sub-cells. As the coupling coefficient $e_{\theta}$ is proportional to the side of the square cells, it is divided by two when considering four sub-cells. The piezoelectric coupling in the macro unit cell in figure 6(a) can thus be represented by the electrical circuit in figure 7 . In fact, this new circuit is made of four times the circuit in figure 5, around 


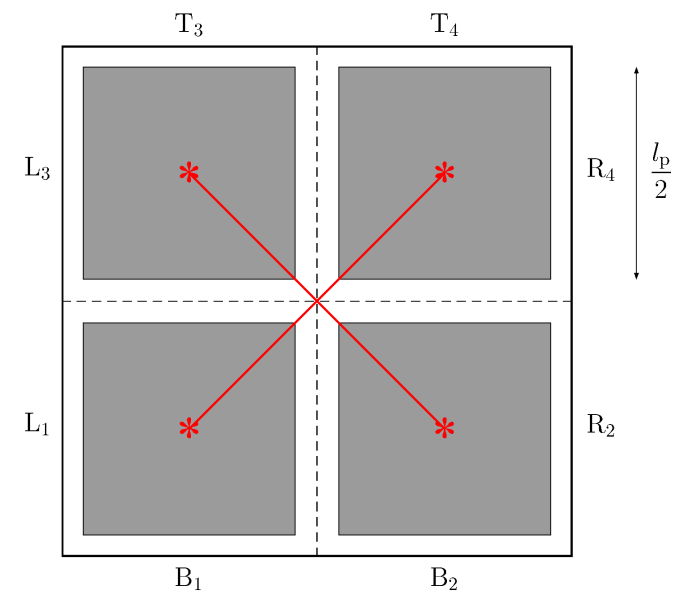

(a)

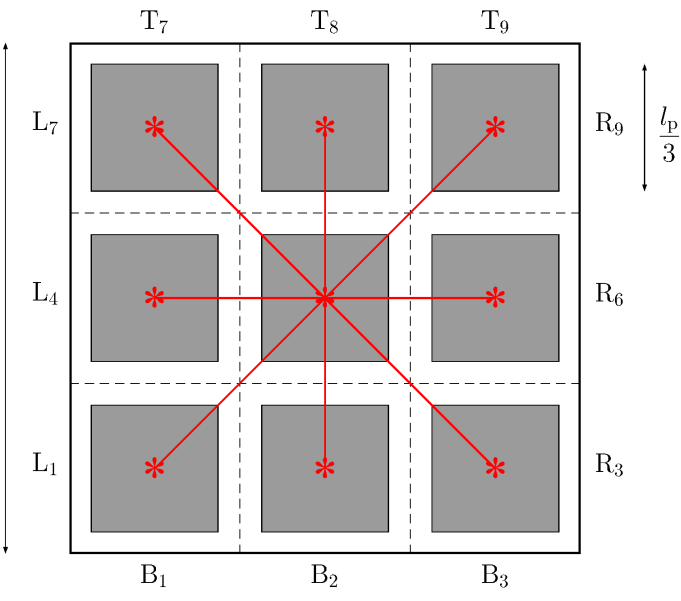

(b)

Figure 6: Macro unit cells: (a) With 4 sub-cells. (b) With 9 sub-cells.

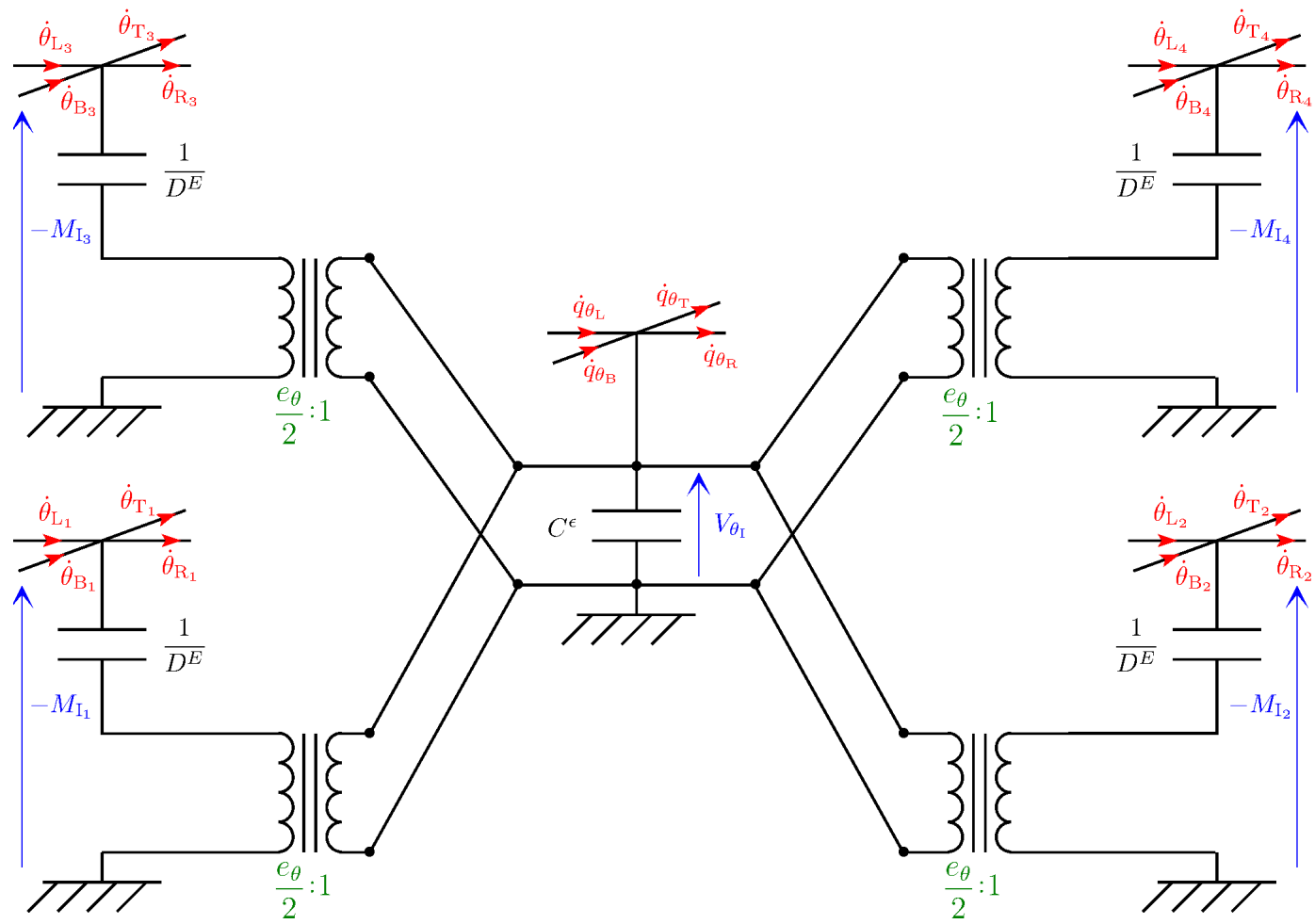

Figure 7: Model of the piezoelectric coupling for a macro unit cell with 4 sub-cells.

a single global capacitance $C^{\varepsilon}$. The numbering of the electrical variables refers to figure $6(\mathrm{a})$, where the side coordinates are written explicitly. In the end, we would get a macro unit cell with a number of mechanical variables that has been doubled. The element matrix $\boldsymbol{D}_{\boldsymbol{c}}^{\mathrm{e}}$ in equation (27) would thus be a $24 \times 24$ matrix.

Depending on the considered wavelength, the macro unit cell in figure 6(a) may still require a finer mesh to generate a suitable approximation of the continuous plate. With square elements, a refined model involves 9 sub-cells instead of 4 , which leads to the macro unit cell represented in figure 6(b). A similar electrical circuit as in figure 7 could be drawn with 9 transformers connected to a single capacitor. From the whole set of equations defining the electrical unit cell and the 9 mechanical sub-cells, a $32 \times 32$ element matrix $\boldsymbol{D}_{\boldsymbol{c}}^{\mathrm{e}}$ is obtained. Here, the force vectors on the sides of 
the macro unit cell are

$$
\begin{aligned}
& \boldsymbol{F}_{\mathrm{B}}^{\mathrm{e}}=\left[-Q_{\mathrm{B}_{1}}-M_{\mathrm{B}_{1}}-Q_{\mathrm{B}_{2}}-M_{\mathrm{B}_{2}}-Q_{\mathrm{B}_{3}}-M_{\mathrm{B}_{3}} V_{w_{\mathrm{B}}} V_{\theta_{\mathrm{B}}}\right]^{T} \\
& \boldsymbol{F}_{\mathrm{L}}^{\mathrm{e}}=\left[-Q_{\mathrm{L}_{1}}-M_{\mathrm{L}_{1}}-Q_{\mathrm{L}_{4}}-M_{\mathrm{L}_{4}}-Q_{\mathrm{L}_{7}}-M_{\mathrm{L}_{7}} V_{w_{\mathrm{L}}} V_{\theta_{\mathrm{L}}}\right]^{T} \\
& \boldsymbol{F}_{\mathrm{R}}^{\mathrm{e}}=\left[Q_{\mathrm{R}_{3}} M_{\mathrm{R}_{3}} Q_{\mathrm{R}_{6}} M_{\mathrm{R}_{6}} Q_{\mathrm{R}_{9}} M_{\mathrm{R}_{9}}-V_{w_{\mathrm{R}}}-V_{\theta_{\mathrm{R}}}\right]^{T} \\
& \boldsymbol{F}_{\mathrm{T}}^{\mathrm{e}}=\left[Q_{\mathrm{T}_{7}} M_{\mathrm{T}_{7}} Q_{\mathrm{T}_{8}} M_{\mathrm{T}_{8}} Q_{\mathrm{T}_{9}} M_{\mathrm{T}_{9}}-V_{w \mathrm{~T}}-V_{\theta_{\mathrm{T}}}\right]^{T} \\
& \boldsymbol{q}_{\mathrm{B}}^{\mathrm{e}}=\left[\begin{array}{llllllll}
W_{\mathrm{B}_{1}} & \theta_{\mathrm{B}_{1}} & W_{\mathrm{B}_{2}} & \theta_{\mathrm{B}_{2}} & W_{\mathrm{B}_{3}} & \theta_{\mathrm{B}_{3}} & q_{w_{\mathrm{B}}} & q_{\theta_{\mathrm{B}}}
\end{array}\right]^{T} \\
& \boldsymbol{q}_{\mathrm{L}}^{\mathrm{e}}=\left[\begin{array}{llllllll}
W_{\mathrm{L}_{1}} & \theta_{\mathrm{L}_{1}} & W_{\mathrm{L}_{4}} & \theta_{\mathrm{L}_{4}} & W_{\mathrm{L}_{7}} & \theta_{\mathrm{L}_{7}} & q_{w_{\mathrm{L}}} & q_{\theta_{\mathrm{L}}}
\end{array}\right]^{T} \\
& \boldsymbol{q}_{\mathrm{R}}^{\mathrm{e}}=\left[\begin{array}{llllllll}
W_{\mathrm{R}_{3}} & \theta_{\mathrm{R}_{3}} & W_{\mathrm{R}_{6}} & \theta_{\mathrm{R}_{6}} & W_{\mathrm{R}_{9}} & \theta_{\mathrm{R}_{9}} & q_{w_{\mathrm{R}}} & q_{\theta_{\mathrm{R}}}
\end{array}\right]_{T}^{T} \\
& \boldsymbol{q}_{\mathrm{T}}^{\mathrm{e}}=\left[\begin{array}{llllllll}
W_{\mathrm{T}_{7}} & \theta_{\mathrm{T}_{7}} & W_{\mathrm{T}_{8}} & \theta_{\mathrm{T}_{8}} & W_{\mathrm{T}_{9}} & \theta_{\mathrm{T}_{9}} & q_{w_{\mathrm{T}}} & q_{\theta_{\mathrm{T}}}
\end{array}\right]^{T}
\end{aligned}
$$

where the numbering refers to figure $6(\mathrm{~b})$. This last macro unit cell based on a $32 \times 32$ element "dynamic stiffness matrix" is finally the one that is implemented in the following comparisons with experimental results in order to ensure a minimum of 10 sub-cells per wavelength in the mechanical domain.

\section{Experimental setup}

The experimental setup is made of a clamped aluminum plate covered with an array of 20 piezoelectric patches. Those are glued onto the plate by using a vacuum bagging process. Then, the analogous network is assembled with passive electrical components, as inductors and transformers, whose number depends on the number of piezoelectric patches.

\subsection{Clamped plate}

The mechanical structure to control is a clamped aluminum plate, whose dimensions and material properties are listed in table 1 . With the considered geometry, the plate corresponds to a set of $5 \times 4$ identical squares of side $80 \mathrm{~mm}$. The clamping frame is made of square aluminum bars reinforced with steel angle channels, as seen in figures 8(a) and $8(\mathrm{~b})$. Two rows of bolts are equally tightened with a torque wrench to ensure zero deflection and zero slope boundary conditions. A white noise excitation is generated with a shaker which is placed at a position $x=80 \mathrm{~mm}$ and $y=120 \mathrm{~mm}$ from the bottom left hand corner of the plate. The input force is measured with a force sensor placed between the shaker and the plate, while the transverse velocity field is measured with a laser scanning laser vibrometer. The force signal is then processed together with the velocity signal to compute velocity frequency response functions (FRFs).

Note that the considered bolted frame offers an adequate approximation of clamped boundary conditions up to $500 \mathrm{~Hz}$. Above this frequency, the two rows of bolts still lead to a satisfactory clamping but the frame and its connections to the table are not

Table 1: Geometry and material properties.

Plate (Al 1050) Patches (PZT-5H)

\begin{tabular}{lll}
\hline Length & $l_{x}=400 \mathrm{~mm}$ & $l_{\mathrm{p}}=72.4 \mathrm{~mm}$ \\
Width & $l_{y}=320 \mathrm{~mm}$ & $l_{\mathrm{p}}=72.4 \mathrm{~mm}$ \\
Thickness & $h_{\mathrm{s}}=1.9 \mathrm{~mm}$ & $h_{\mathrm{p}}=0.27 \mathrm{~mm}$ \\
\hline Density & $\rho_{\mathrm{S}}=2700 \mathrm{~kg} / \mathrm{m}^{3}$ & $\rho_{\mathrm{p}}=7800 \mathrm{~kg} / \mathrm{m}^{3}$ \\
Young's modulus & $Y_{\mathrm{S}}=69 \mathrm{GPa}$ & $1 / s_{11}^{E}=62 \mathrm{GPa}$ \\
Charge constant & - & $d_{31}=-320 \mathrm{pC} / \mathrm{N}$ \\
Permittivity & - & $\epsilon_{33}^{\sigma}=33.6 \mathrm{nF} / \mathrm{m}$ \\
\hline
\end{tabular}

stiff enough, which generates undesired low-frequency frame modes. So, the velocity FRFs are measured over a frequency range spanning from $50 \mathrm{~Hz}$ to $500 \mathrm{~Hz}$.

\subsection{Array of piezoelectric patches}

The aluminum plate is covered with an array of 20 square PZT-5H patches, whose dimensions and properties are given in table 1. As performed by Anton et al. [38], the patches are glued onto the plate by using a vacuum bagging process, which allows reducing the thickness of the bonding layer. We chose the 3M DP460 two-part epoxy adhesive for its high shear strength and its adequate working life. The epoxy adhesive is applied on one side of the piezoelectric patches before their positioning onto the plate. The plate is then placed in the vacuum bag shown in figure 9(a). After 24 hours, we obtain the functional piezoelectric plate in figure $9(\mathrm{~b})$, which can be inserted into its clamping frame.

The addition of piezoelectric patches does not strongly influence the modal distribution of the plate because both stiffness and mass are increased. Compared to a case without any patch, bonding opencircuited patches increases the natural frequency of the first mode by only $1.5 \%$. Furthermore, we can evaluate the difference in terms of natural frequencies when the patches are short- or open-circuited. As a reference, the first mode of the plate is around $140 \mathrm{~Hz}$ with open-circuited patches and this frequency is decreased by $3.5 \%$ once the patches are shortcircuited. By comparing this experimental result with simulations from the model developed in section 3 , the global coupling coefficient appearing in equation (26) is updated to $e_{\theta}=1.4 \times 10^{-3} \mathrm{~N} \cdot \mathrm{m} / \mathrm{V}$.

With the vacuum bagging process, the bonding layer is actually so thin that we get a direct contact between the plate and at least one corner of the piezoelectric patches, which creates a short circuit even with a "nonconductive" adhesive. The plate and its clamping frame are thus defined as the ground of the electrical circuit when considering the coupling to 


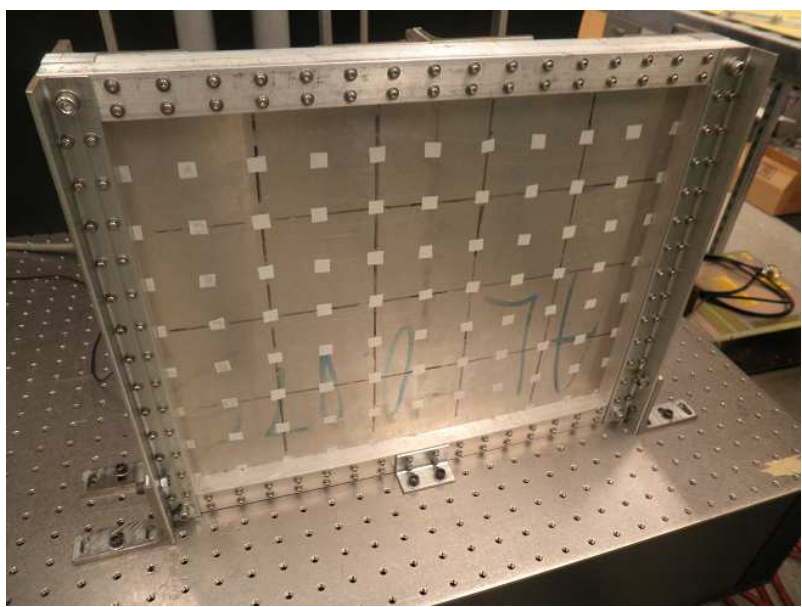

(a)

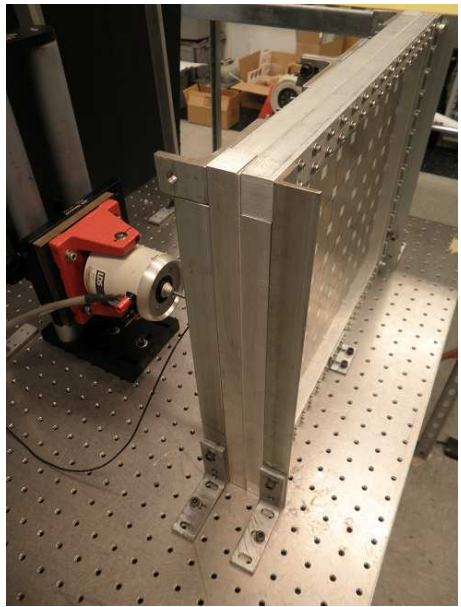

(b)

Figure 8: Clamped aluminum plate: (a) Front of the plate with reflective tape for velocity measurement with a scanning laser vibrometer. (b) Shaker exciting the back of the plate.

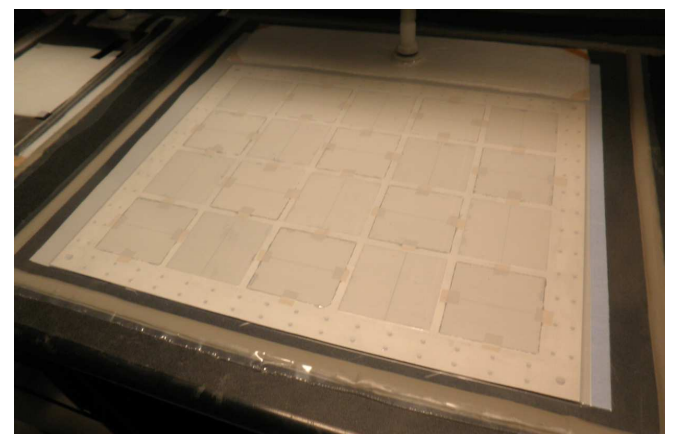

(a)

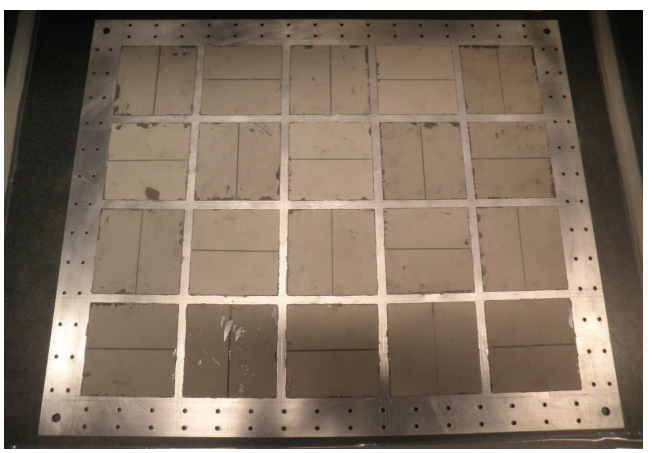

(b)

Figure 9: Vacuum bonding process: (a) Plate in the vacuum bag. (b) Plate covered with piezoelectric patches after vacuum bonding.

an electrical network. The piezoelectric capacitance is evaluated by direct measurement on the patches at the corners of the clamped plate. There, the boundary conditions naturally constrain the transverse displacement and it is found that the capacitance is $C^{\varepsilon}=340 \mathrm{nF}$.

\subsection{Analogous electrical network}

The analogue of a clamped plate is built by referring to the electrical unit cell in figure 3. No capacitors $C_{0} / 2$ are required because this element has only been introduced to allow numerical simulations. Moreover, we do not need to consider external capacitors $C$ because the electrical network is intended for coupling to the plate through the array of piezoelectric patches that offer a capacitance $C^{\varepsilon}$. The analogous electrical network is thus implemented by connecting together $5 \times 4$ identical unit cells, which corresponds to the number of piezoelectric patches.

The electrical states along the edges of the network have to satisfy an analogy with mechanical boundary conditions. As we focus on the analogue of a clamped plate, the zero-displacement and zero-angle boundary conditions, $W=0$ and $\theta=0$ leads to $q_{w}=0$ and $q_{\theta}=0$ along the boundaries of the network. This means that we have to leave the corresponding electrical ports open-circuited. Then, no current flows through the inductors and transformers along the four edges of the network. Those components are therefore not required and can be removed from the network. Furthermore, the pairs of $\hat{a} / 2$ ratio transformers can be replaced by single transformers of ratio $\hat{a}$. For practical reasons related to the design of the magnetic components, a ratio $\hat{a}=4$ has been chosen. In the end, the network is assembled with 17 transformers of ratio 4:1, 6 transformers of ratio $1: 1$ and 6 inductors $L=0.9 \mathrm{H}$. This value comes from the modal coupling condition in equation (7) with the bending stiffness 


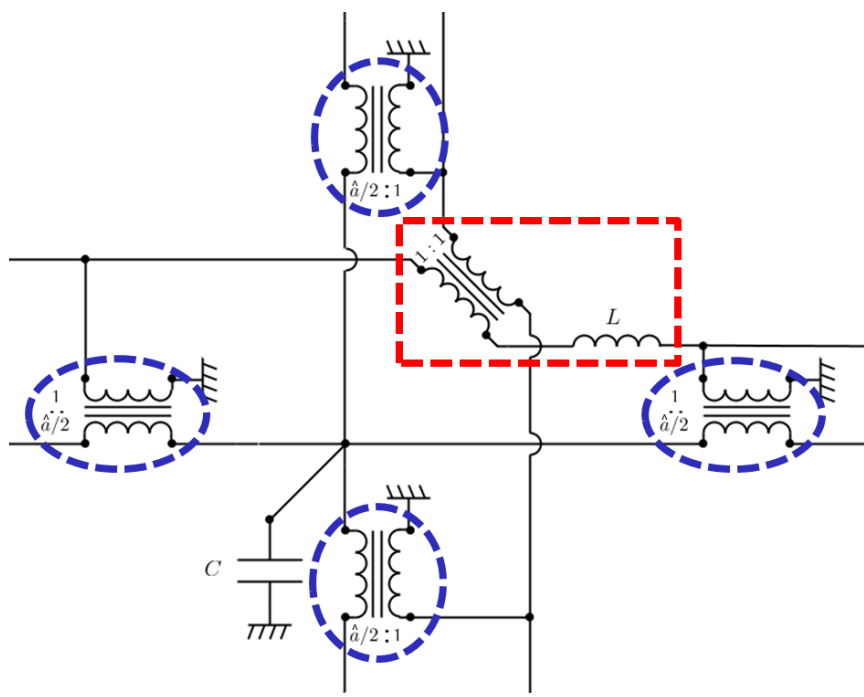

(a)

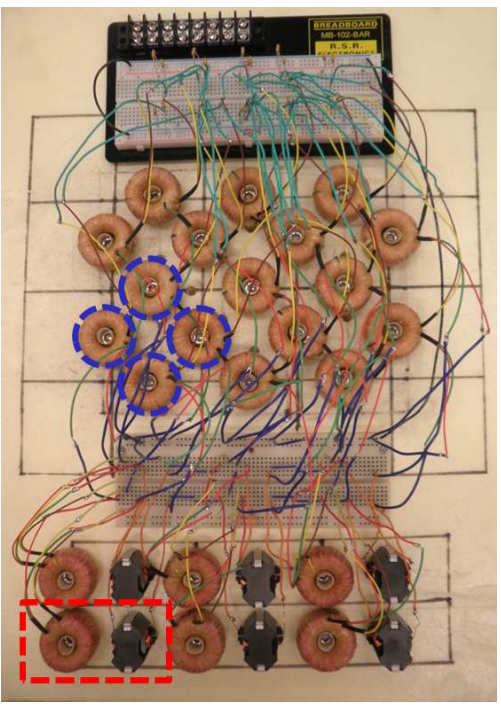

(b)

Figure 10: Implementation of the analogous network: (a) Electrical unit cell. (b) Network made of $5 \times 4$ unit cells.

$D^{E}$ and the capacitance $C^{\varepsilon}$. Under this condition, an infinite number of unit cells would lead to identical modal properties for the plate and for its analogous electrical network. Practical applications does not offer an infinite number of unit cells, but still, the inductance is tuned for optimal damping around at least the first mode of the plate.

The inductors are made by winding copper wire around type RM ferrites [39, 40]. Direct measurements on the inductors gives a series resistance $R_{\mathrm{S}}^{L}=2.6 \Omega$ and a parallel resistance $R_{\mathrm{p}}^{L}=200 \mathrm{k} \Omega$. Concerning the transformers, they are wound around nanocrystalline toroidal cores $[39,40]$. The 4:1 transformers are made with 3000 turns on the primary winding and 750 turns on the secondary winding, which gives a total winding resistance $R_{\mathrm{S}}^{T}=353 \Omega$. For the 1:1 transformers, two windings of 750 turns leads to a winding resistance $R_{\mathrm{S}}^{T^{*}}=21 \Omega$. The positioning of the electrical components is presented through figures 10(a) and 10(b), where we note the frames with dashed lines that indicate the correspondence between the topology of the unit cell and the actual electrical network.

\section{Results and discussion}

Experiments with short-circuited patches give a reference case when no control occurs. The analogous electrical network is then coupled to the clamped plate through the piezoelectric array. The electrical resonances strongly influence the dynamic response of the plate, which is optimized by adjusting the inductance and resistance values.

\subsection{Modal analysis with short-circuited patches}

The first experimental results focus on the modal analysis of the clamped plate when the piezoelectric patches are short-circuited. The scanning vibrometer is used to get experimental operating deflection shapes. For the FRF, the measurement point for the velocity corresponds to the position $x=120 \mathrm{~mm}$ and $y=120$ $\mathrm{mm}$ from the bottom left hand corner of the plate. Figure 11 shows four resonances over the considered frequency range. Actually, the $4^{\text {th }}$ resonance is the result of a combination of the $4^{\text {th }}$ and $5^{\text {th }}$ modes that exhibit close natural frequencies.

The coupled models developed in section 3 can be used for a comparison with the experimental results involving short-circuited patches by setting the coupling coefficient $e_{\theta}$ to zero. The bending stiffness $D^{E}$ and the mass $m$ of the unit cell are calculated from its geometry and material properties. The model involving a macro unit cell with 9 sub-cells is then considered. This generates a total of $15 \times 12$ sub-cells, which ensures on a minimum of 10 mechanical unit cells per wavelength for the first five plate modes of the clamped plate. When comparing experimental and numerical FRFs, we have noticed the non-negligible influence of the mass $\tilde{m}=7.6 \mathrm{~g}$ of the element which is added between the force sensor and the plate for connecting one to the other. Consequently, there is a difference between the simulated force $F_{\text {sim }}$ acting on the plate and the force $F_{\exp }$ measured by the force sensor. If $F_{\exp }$ is the force applied by the force sensor to the mass $\tilde{m}$ and $F_{\text {sim }}$ is the force applied to the 


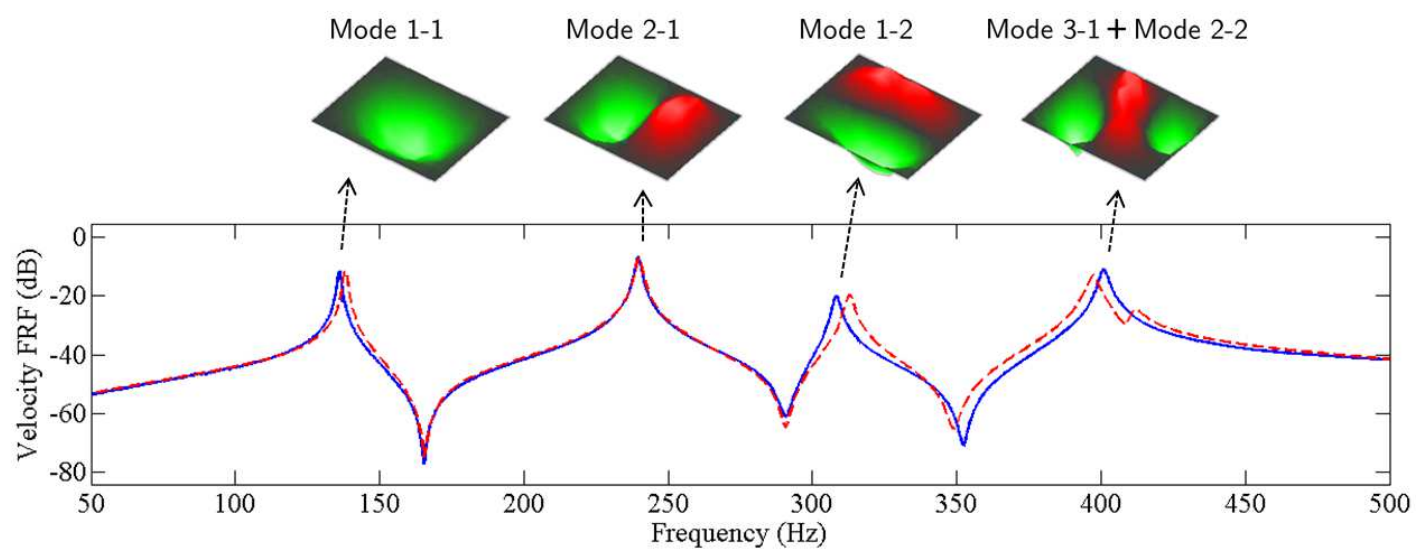

Figure 11: Experimental operating deflection shapes with respect to the velocity FRF - (-) experimental FRF, $(--)$ simulated FRF computed with $15 \times 12$ sub-cells.

plate

$F_{\exp }-F_{\text {sim }}=-\omega^{2} \tilde{m} \tilde{W}$,

where $\tilde{W}$ is the displacement of the plate that has been computed at the point of excitation. Consequently, if a displacement field has first been computed with $F_{\text {sim }}=1$, it has to be divided by $1-\omega^{2} \tilde{m} \tilde{W}$ to find the actual values taking into account the added mass. This improves the correlation between numerical and experimental results but we still note that neither mass of bonding layers and electrical wires nor eventual softening effect due to imperfect clamping conditions are considered in the model. For this reason, the parameter related to the mass of the unit cell might need to be updated. This finally led to a negligible $1 \%$ increase of $m$ when considering the present setup.

Another model update concerns structural damping. A viscous damping applied to the transverse displacement is combined to a viscous damping applied to the stiffness. This corresponds to the use of an equivalent complex density as $\rho(1-4.0 \mathrm{j} / \omega)$ and an equivalent complex Young's modulus as $Y\left(1+3.3 \times 10^{-6} \mathrm{j} \omega\right)$, whose damping coefficients were determined by comparison with the experimental results. This damping model approximates the maxima of the velocity FRF below $500 \mathrm{~Hz}$. Indeed, figure 11 shows that the numerical results are in good agreement with the experimental ones. The main difference remains in the fact that the $4^{\text {th }}$ and $5^{\text {th }}$ modes can be distinguished on the simulated FRF. Yet, we note that refining the mesh does not clearly improve the correlation because it cannot overcome the bias introduced by the non-ideal clamped boundary conditions in the experiments.

\subsection{Multimodal coupling}

After preliminary experiments involving short-circuited patches, i.e. no piezoelectric coupling, the plate is cou-

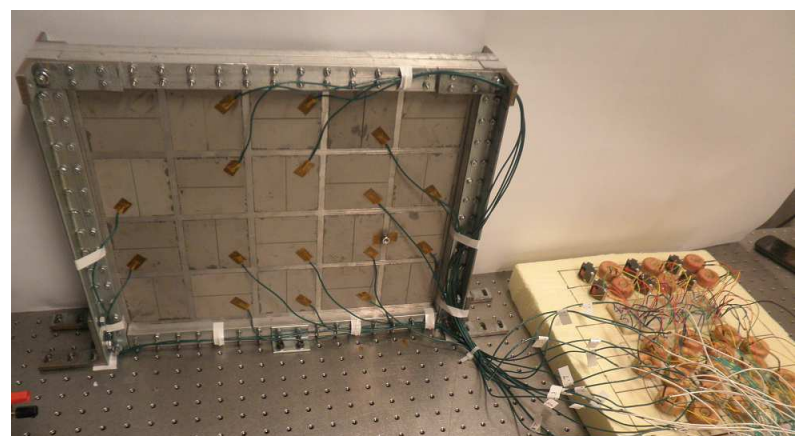

Figure 12: Clamped aluminum plate covered with piezoelectric patches connected to the multi-resonant network.

pled to it electrical network in figure 10(b). According to the discrete network topology obtained for a clamped plate, the piezoelectric patches on the corners do not play any role. Only 16 of the 20 patches are thus connected to the electrical network for the present application. The resulting coupled system is presented in figure 12. Once the network is engaged, figure 13 shows that the coupling provides a strong modification of the modal behavior of the plate. At $140 \mathrm{~Hz}$ and $230 \mathrm{~Hz}$, we remark local minima surrounded by two local maxima. This is a characteristic of an underdamped tuned mass control, which is here generated by the multi-resonant, spatially-distributed network. This confirms that the piezoelectric coupling of a $2 \mathrm{D}$ mechanical structure to its electrical analogue leads to a passive control that acts as a multimodal tuned mass damping, similarly to what has been implemented previously for the control of 1D structures $[29,30]$.

The vibration reduction is also significant for the highest modes but the tuned mass effect is less obvious. Actually, the network does not offer a tuning that is 


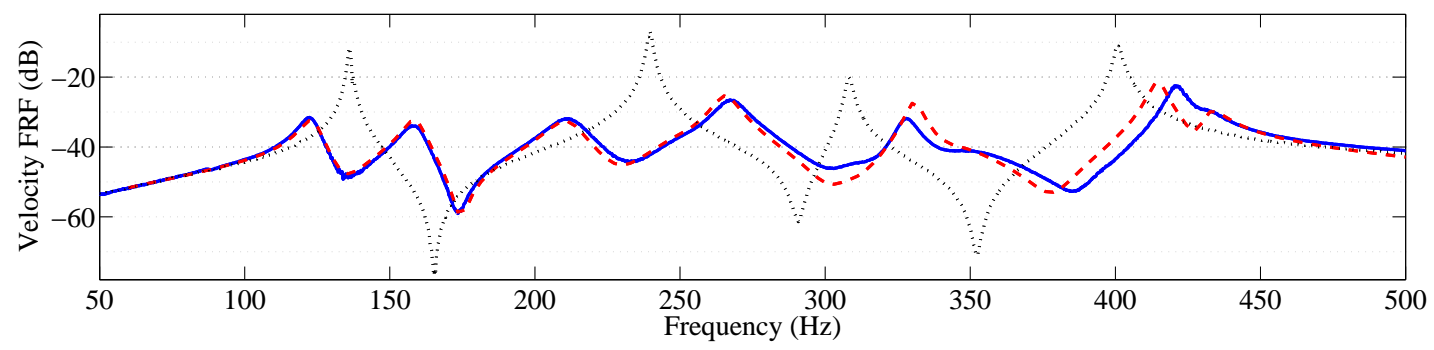

Figure 13: Influence of the electrical network on the plate velocity FRF $-(\cdots)$ experimental FRF with shortcircuited patches, (-) experimental FRF with $L=0.9 \mathrm{H}$ and $R_{\mathrm{S}}^{+}=0 \Omega,(--)$ simulated FRF with $L=0.9 \mathrm{H}$ and $R_{\mathrm{S}}^{+}=0 \Omega$.

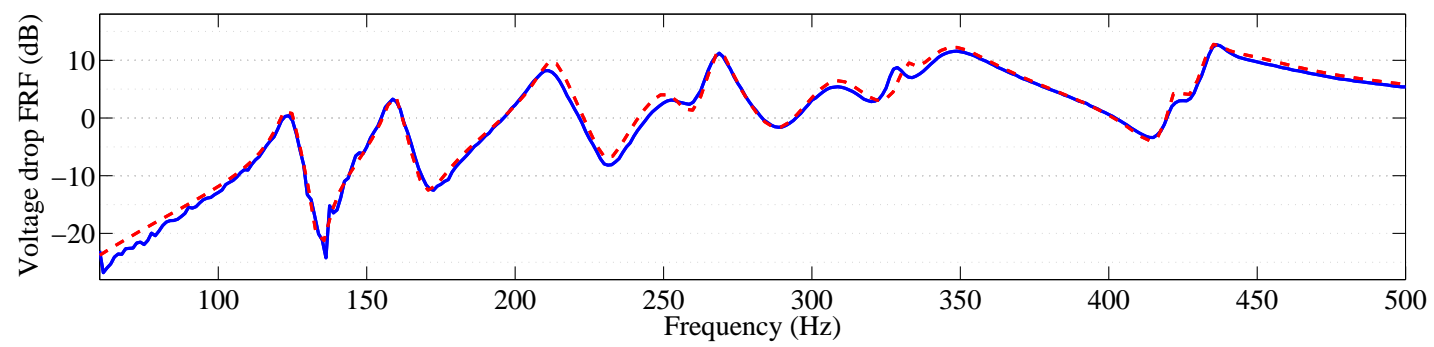

Figure 14: Voltage frequency response function - $(-)$ experimental FRF with $L=0.9 \mathrm{H}$ and $R_{\mathrm{S}}^{+}=0 \Omega$, (--) simulated FRF with $L=0.9 \mathrm{H}$ and $R_{\mathrm{S}}^{+}=0 \Omega$.

optimized for all the modes simultaneously. The exact analogue of a continuous structure cannot be obtained with a set of discrete electrical components. Therefore, our discrete network only offers an approximation of the continuous plate behavior at low frequency. The lower electrical resonances thus occur at frequencies that are close to the corresponding mechanical resonances but for higher modes the frequency error becomes non-negligible. Nevertheless, it would be possible to more closely match the resonances of the plate with the same network topology by increasing the number of electrical unit cells.

Considering the comparison between the experimental results and those coming from the model developed in section 3, an adequate correlation is observed in figure 13. Still, we note an increasing discrepancy over the highest frequency range, which is due to two main reasons. First, even if the considered model involves macro unit cells offering $15 \times 12$ sub-cells for the plate, it is still a discrete model that is close to its limits for the highest modes. Second, the approximation of perfect clamped boundary conditions with the actual setup becomes questionable when approaching $500 \mathrm{~Hz}$.

Another solution to validate the numerical model and to verify the tuning of the network is to measure electrical frequency response functions. Those can be obtained from a voltage excitation on a node of the network and a measurement of the voltage drop across an inductor. The measurement and excitation points are the analogues of those defined for the velocity FRFs. The piezoelectric coupling between the network and the plate considerably influences the electrical FRF presented in figure 14. We note a sharp antiresonance around $140 \mathrm{~Hz}$ that characterize the first mode of the plate. This antiresonance illustrates the electromechanical energy transfer and its positioning between two local maxima presenting close ordinates shows that the tuning of the network is adequate $[29,30]$. In the end, the simulation of the electrical FRF is very reliable, which definitely validates the proposed model based on two-dimensional coupling to an analogous network.

\subsection{Tuning of the electrical network}

Depending on the application, the electrical network can be tuned in different ways. For example, if a control is required at one specific frequency which is near a mechanical resonance, the inductors can be adjusted in order to place the corresponding electrical resonance around the target frequency. This generates an antiresonance, as seen in figure 13 around the first mode of the plate. Then, the internal damping in the components must be minimized in order to increase the depth of the antiresonance.

On the other hand, a control over a broader frequency range requires the introduction of additional damping in the network. Indeed, it is shown in 


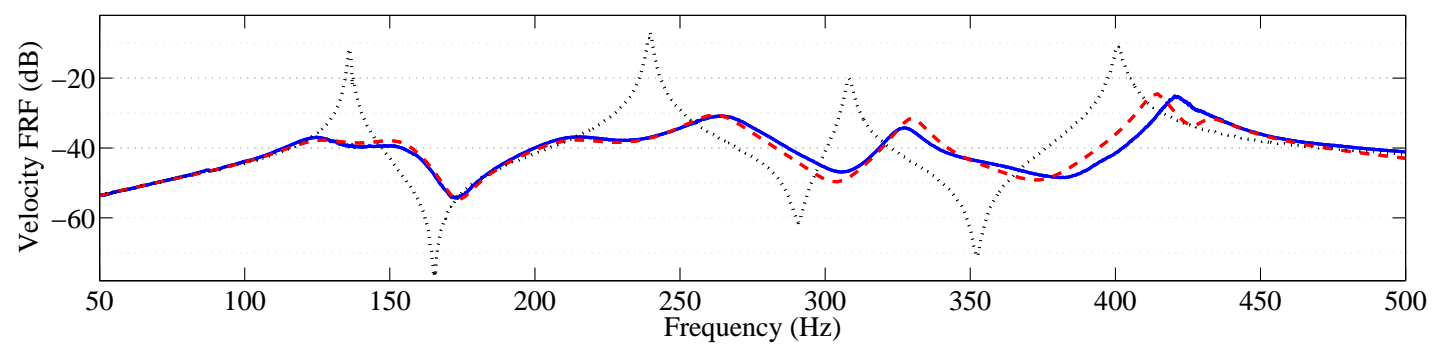

Figure 15: Addition of damping in the electrical network - $(\cdots)$ experimental FRF with short-circuited patches, $(-)$ experimental FRF with $L=0.9 \mathrm{H}$ and $R_{\mathrm{S}}^{+}=180 \Omega,(--)$ simulated FRF with $L=0.9 \mathrm{H}$ and $R_{\mathrm{S}}^{+}=180 \Omega$.

figure 15 that $180 \Omega$ resistors in series with the 6 inductors flatten the FRF. Note that the addition of resistance does not necessarily increase the number of components as it can be taken into account when designing the inductors. A vibration reduction around $25 \mathrm{~dB}$ is obtained for the first two modes of the plate. Focusing on passive control solutions, such damping performances could not be reached with multiple-mode shunt circuits $[9,8,10]$, which require external capacitors that decrease the electromechanical coupling [17].

The comparison between figures 13 and 15 shows that the introduction of resistors mainly affects the lowest modes. Again, this is due to the fact that the discrete electrical network yields lower coupling at higher frequencies. With the discrete network, a solution for a vibration reduction over a broader frequency range is to detune the first modes to better tune higher modes. This strategy is presented in figure 16 , where the previous case involving a network with $0.9 \mathrm{H}$ inductors and $180 \Omega$ series resistors is compared to a second case with $0.7 \mathrm{H}$ inductors and the same $180 \Omega$ resistors. The second inductance value has been obtained from a $\mathcal{H}_{\infty}$ optimization, which minimizes the maximum of the velocity FRF over the $50 \mathrm{~Hz}$ to $500 \mathrm{~Hz}$ frequency range:

$$
\mathcal{H}_{\infty}=\min _{L}\left(\max _{f}[\operatorname{FRF}(f, L)]\right),
$$

where $f \in[50 \mathrm{~Hz}, 500 \mathrm{~Hz}]$. By decreasing the inductance, the electrical resonances move to higher frequencies. In this case, we remark that the second electrical resonance is moved from a frequency below the second mechanical resonance to a frequency above it. Even if the modification of the tuning is not beneficial for the first mode, the maximum of the amplitude over the frequency range of interest is minimized. The decrease of the inductance moves the highest electrical resonances closer to their mechanical analogues, which clearly reduces the amplitude of the FRF around its upper frequency range. In the end, the vibration reduction is above $20 \mathrm{~dB}$ for the first five modes of the plate when compared to the short- circuited case. This shows that the analogous coupling offers an efficient broadband control, even with a coarse discretization of the electrical network.

\section{Conclusions}

A distributed control strategy has been implemented by means of an array of piezoelectric patches that are interconnected through an analogous electrical network. For a square plate unit cell, a finite difference method applied to the Kirchhoff-Love theory gives a discrete model that is converted into its direct electrical analogue. Focusing on clamped boundary conditions, the novel analogous topology is implemented with a set of purely passive electrical components, which proves that the control solution does not require any power supply. A significant modification of the mechanical response is observed once the passive electrical network is coupled to the considered clamped plate. To the best of our knowledge, this work presents the first experiments involving the coupling of a plate to an analogous electrical network. Because the network is discrete and does not offer a large number of unit cells, an optimal coupling can only be achieved around the lowest modes the continuous plate. Nevertheless, an increase of the number of unit cell would allow a broadband control up to any target frequency, as long as the Kirchhoff-Love assumptions are still valid.

The proposed coupled model based on 2D electromechanical unit cells is also validated from the experiments. We note that the frequency range of interest influences the choice of a suitable macro unit cell. For instance, the macro unit cell offering 4 subcells could have been sufficient in the low-frequency range. On the other hand, a simulation of the present setup above $500 \mathrm{~Hz}$ would require more than 9 sub-cells to maintain a minimum of 10 elements per wavelength. Yet, an increase of the number of sub-cell leads to large symbolic element matrices that can be difficult to handle. A natural perspective thus consists in the definition of a finite element model with adaptive mesh for the mechanical part of the unit cell [41]. 


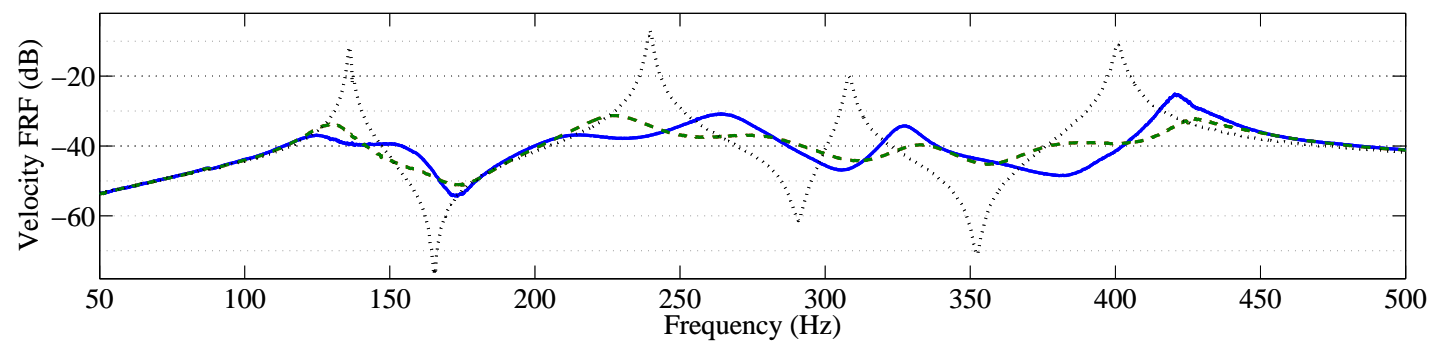

Figure 16: Broadband tuning of the network - ( ..) experimental FRF with short-circuited patches, $(-)$ experimental FRF with $L=0.9 \mathrm{H}$ and $R_{\mathrm{S}}^{+}=180 \Omega,(--)$ experimental FRF with $L=0.7 \mathrm{H}$ and $R_{\mathrm{S}}^{+}=180 \Omega$.

In any case, the present discrete models still offer a suitable approximation of the continuous plate in the low-frequency range and they allow preliminary simulations that are essential for the design of the electrical components. Depending on the application, a suitable tuning of the electrical network can then optimize the vibration amplitude around one specific frequency or over a broad frequency range. When the network is tuned for broadband damping, we get a $20 \mathrm{~dB}$ vibration reduction over the $50 \mathrm{~Hz}$ to $500 \mathrm{~Hz}$ frequency range, which definitely shows the interest of the passive control strategy.

\section{Acknowledgments}

This work was funded by the French Ministry of National Education, Higher Education and Research through a three year scholarship for doctoral studies related to structural damping with piezoelectric devices. Furthermore, the authors want to thank the Fulbright Program, which made possible a ninemonth research visit to the Georgia Institute of Technology. Thanks also go to Stephen Leadenham for the implementation of the vacuum bonding process.

\section{References}

[1] Hagood N and von Flotow A 1991 Journal of Sound and Vibration 146 243-268

[2] Spadoni A, Ruzzene M and Cunefare K A 2009 Journal of Intelligent Material Systems and Structures 20 979-990

[3] Casadei F, Ruzzene M, Dozio L and Cunefare K A 2010 Smart Materials and Structures 19015002

[4] Casadei F, Dozio L, Ruzzene M and Cunefare K A 2010 Journal of Sound and Vibration 329 3632-3646

[5] Chen S, Wang G, Wen J and Wen X 2013 Journal of Sound and Vibration 332 1520-1532

[6] Fleming A J, Behrens S and Moheimani S O R 2003 Smart Materials and Structures $\mathbf{1 2} 57$

[7] Thomas O, Ducarne J and Deü J F 2012 Smart Materials and Structures 21015008

[8] Hollkamp J J 1994 Journal of Intelligent Material Systems and Structures $\mathbf{5} 49-57$

[9] Wu S Y 1998 Journal of Intelligent Material Systems and Structures 9 991-998
[10] Behrens S, Moheimani S and Fleming A 2003 Journal of Sound and Vibration 266 929-942

[11] Collet M, Ouisse M, Ichchou M N and Ohayon R 2012 Smart Materials and Structures 21094002

[12] Casadei F, Beck B S, Cunefare K A and Ruzzene M 2012 Journal of Intelligent Material Systems and Structures 23 1169-1177

[13] Tateo F, Collet M, Ouisse M, Ichchou M, Cunefare K A and Abbe P 2015 Journal of Intelligent Material Systems and Structures 26 952-964

[14] Vidoli S and dell'Isola F 2001 European Journal of Mechanics - A/Solids 20 435-456

[15] Maurini C, dell'Isola F and Del Vescovo D 2004 Mechanical Systems and Signal Processing 18 124-1271

[16] Batra R, dell'Isola F, Vidoli S and Vigilante D 2005 International Journal of Solids and Structures 423115 $-3132$

[17] Giorgio I, Culla A and Del Vescovo D 2008 Archive of Applied Mechanics 79 859-879

[18] dell'Isola F, Porfiri M and Vidoli S 2003 Comptes Rendus Mécanique 331 69-76

[19] Alessandroni S, dell'Isola F and Porfiri M 2002 International Journal of Solids and Structures 39 5295-5324

[20] Porfiri M, dell'Isola F and Frattale Mascioli F M 2004 International Journal of Circuit Theory and Applications 32 167-198

[21] Bloch A 1945 Journal of the Institution of Electrical Engineers - Part I: General 92 157-169

[22] MacNeal R H 1949 The solution of partial differential equations by means of electrical networks Ph.D. thesis URL http://resolver . caltech.edu/CaltechETD: etd-04282004-143609

[23] Benscoter S U and MacNeal R H 1952 Introduction to electrical-circuit analogies for beam analysis Tech. rep. NACA URL http://naca.central.cranfield.ac. uk/report $\cdot$ php?NID $=4941$

[24] Benscoter S U and MacNeal R H 1952 Equivalentplate theory for a straight multicell wing Tech. rep. NACA URL http://naca.central.cranfield.ac.uk/ report . php?NID $=4942$

[25] MacNeal R H 1954 Electrical analogies for stiffened shells with flexible rings Tech. rep. NACA URL http://naca. central. cranfield.ac . uk/report.php?NID $=5940$

[26] Alessandroni S, Andreaus U, dell'Isola F and Porfiri M 2004 European Journal of Mechanics - A/Solids 23 689-702

[27] Alessandroni S, Andreaus U, dell'Isola F and Porfiri M 2005 Computers \& Structures 83 1236-1250

[28] Lossouarn B, Cunefare K A, Aucejo M and Deü J F 2016 Robustness of a multimodal piezoelectric damping involving the electrical analogue of a plate URL http: $/ / \mathrm{dx}$.doi.org/10.1117/12.2218357

[29] Lossouarn B, Aucejo M and Deü J F 2015 Smart Materials 
and Structures 24045018

[30] Lossouarn B, Deü J F and Aucejo M 2015 Smart Materials and Structures $\mathbf{2 4} 115037$

[31] Timoshenko S P 1940 Theory of plates and shells (McGrawHill)

[32] Cho Y S, Pak Y, Han C S and Ha S K 2000 Sensors and Actuators A: Physical 84 140-148

[33] Beranek L L 1954 Acoustics (McGraw-Hill)

[34] ANSI/IEEE 1988 ANSI/IEEE Std 176-1987

[35] Deraemaeker A, Nasser H, Benjeddou A and Preumont A 2009 Journal of Intelligent Material Systems and Structures 20 1475-1482

[36] Ducarne J, Thomas O and Deü J F 2012 Journal of Sound and Vibration 331 3286-3303

[37] Maurini C, Pouget J and dell'Isola F 2006 Computers 83 Structures 841438 - 1458

[38] Anton S R, Erturk A and Inman D J 2010 Smart Materials and Structures 19115021

[39] McLyman C W T 2004 Transformer and Inductor Design Handbook (CRC Press)

[40] Multon B 2015 Composants passifs de l'électronique de puissance (magnétiques et capacitifs) Lecture URL https://cel.archives-ouvertes.fr/cel-01246811

[41] Thomas O, Deü J F and Ducarne J 2009 International Journal for Numerical Methods in Engineering 80 235268 